\title{
Multi-functionality of the few: current and past uses of wild plants for food and healing in Liubań region, Belarus
}

Renata Sõukand ${ }^{1 *}$, Yanina Hrynevich², Iryna Vasilyeva², Julia Prakofjewa², Yuriy Vnukovich², Jury Paciupa², Aliaksei Hlushko², Yana Knureva², Yulia Litvinava ${ }^{3}$, Siarhei Vyskvarka ${ }^{4}$, Hanna Silivonchyk ${ }^{5}$, Alena Paulava², Mare Kõiva ${ }^{1}$ and Raivo Kalle ${ }^{1}$

\begin{abstract}
Background: This study examined the use of wild plants in the food, medicinal and veterinary areas within a small territory limited to one village council in the Liubań district of Belarus. The objectives of the research were to document the current and past uses of wild plants in this region for food and human/animal medication; to analyse the food, medicinal and veterinary areas in the context of wild plants; and to qualitatively compare the results with relevant publications concerning the wild food plants of Belarus.

Methods: Fieldwork was carried out as a practical part of a development cooperation project in May 2016 in 11 villages of the Liubań district. One hundred thirty-four respondents were selected randomly. Information about local uses of wild plants was obtained via semi-structured interviews and the folk-history method. Interview records were digitalized and the data structured in Detailed Use Records (DUR), which were divided into food, medicinal and veterinary areas and then analysed to ascertain local perceptions.

Results: A total of 2252 DUR of wild plants were recorded. Eighty-eight wild plant taxa belonging to 45 plant families were used across all three areas. Of these, 58 taxa were used in the food, 74 in the medicinal and 23 in the veterinary areas. A relatively high percentage of the taxa were used in both the food and medicinal areas (55\%) and an even greater percentage in both the medicinal and veterinary areas (87\%). Comparison with earlier research on wild food plants shows the considerable difference among seldom-mentioned taxa or uses, showing possible regional differences despite the homogenization of the population during the Soviet era.

Conclusions: As the majority of taxa with overlapping uses belonged to the most utilized plants, there appears to be clear a tendency to use plants in several different areas once they are brought into the home. This may be due to the need to maximize the versatility of limited resources. While the number of wild taxa used is relatively high, the mean number of taxa used per person is quite low, which indicates the relatively minor importance of wild plants in the respective areas in the study region. The low importance of snacks signals that unintended contact with nature has been lost.
\end{abstract}

Keywords: Belarus, Wild plants, Food plants, Medicinal plants, Food-medicine, Local knowledge, Ethnobotany, Ethnopharmacology, Ethnoveterinary, Liubań

\footnotetext{
* Correspondence: renata@folklore.ee; renata.herba@gmail.com

${ }^{1}$ Estonian Literary Museum, Vanemuise 42, Tartu 51003, Estonia

Full list of author information is available at the end of the article
} 


\section{Background}

Life in present-day, highly literate Europe is rapidly changing. This affects local practices in the use of wild plants for food and healing in both people and animals, which is part of the Local Ecological Knowledge, crucial for sustaining human life on earth. Therefore, an increase of interest in documenting such knowledge and understanding the changes it undergoes is well justified. The classical studies by Etkin [1, 2] and Pieroni and Price [3], as well as more recent field examples in Europe [4-6], have demonstrated either a dual perception and function of food and medicinal uses and even their co-evolution, or a remarkable overlap in the nomenclature of species used simultaneously in the food and medicinal areas. Thus, the need to look at the uses of wild plants from both perspectives, food and medicine, is also warranted.

We can assume that the cultivation of plants brings them closer to people; the use of cultivated plants may increase as people have fewer encounters with the wild (for example [7]). Wild plants, in contrast, become more distant and less known, as a result of habitat loss, changes in the paths of human movement and the lessened need for wild supplies, as well as changes in cultural attitudes to wild foods or tastes $[8,9]$. Simple logic will say that once the wild plant is brought home, it will be used for as many purposes as possible, for as long as the amounts gathered allow.

Luczaj et al. [10] referred to Belarus as terra incognita with respect to modern ethnobotany. Indeed, the majority of the information gathered about ethnomedicinal and food uses of wild plants originates from rather distant times. To date [10], remains the only comprehensive publication on the use of wild food plants in both modern and historical contexts in the territory of Belarus. The first records on traditional medicine in Belarus date to the late XVIII-early XIX centuries, which were included in descriptions of rituals and daily life of the local population. In general, the attention of researchers has been focused on the study of ways to treat the causes of diseases and their prevention. For example, the work titled "Description of the Barysaŭ district" by E. Tyszkiewicz provided a comprehensive list of plants (with local names) with their medicinal (also emic) purpose and usage both in the past and at the time of publication [11]. Throughout the XIX and early XX century a systematic collection and publication of ethnomedicinal field data was conducted for Belarus by numerous authors (for example YE. Romanov [12, 13], N. Nikiforovskiy [1416], M. Federowski [17, 18] P. Shein [19, 20], V. Dobrovol'skiy [21] A. Bahdanovič [22], F. Wereńko [23], N. Yanchuk [24]). The main attention of these researchers was focused on documenting charms, information about the people involved in treatment and the variety of used remedies, including those involving plants. The unique information regarding the local names of plants of the Hrodna region and their medicinal properties are presented in the publication by E. Orzeszkowa [25]. Significant contribution to the systematization of the traditional concepts of Belarusians about the causes and origins of diseases and their treatment were made by Polish (K. Moszyński [26], Cz. Pietkiewicz [27], etc.) and Russian (D. Zelenin [28], G. Popov [29], etc.) scientists. During the Soviet era field research on traditional medicine passed out of scientific interest. As was common within other Soviet regions only a few "critical analyses" of ethnographic data from this period were published (Mińko $[30,31])$, exploring methods of treatment, including the use of herbal medicine and magic. Recently, Belarusian folklorists have been actively studying the semiotic status and symbolic image of plants in traditional culture (I. Šved [32]), non-plant traditional medicine as part of the traditional culture (T. Valodzina [3337]) and ethnographic classification of methods of treatments (U. Lobač [38] and U. Filipienka [39]). However, thus far no comprehensive and complex approach to the regional use of wild plants in Belarus has been published.

Therefore, in this study we decided to address one specific well-defined area of wild plants used for food and medicine pertaining to both humans and other animals within a little-studied territory. While classically such ethnobotanical fieldwork concentrates on food and medicinal uses of plants only, we choose to also include the veterinary area as domesticated animals are still kept in the researched region; and due to the widespread medical veterinary intervention this area is too small to be examined separately. The specific aims of this study are: 1) to document the current and past uses of wild plants in the Liubań district for food and human/animal medication; 2) to analyse the food, medicinal and veterinary areas in the context of wild plants; and 3) to qualitatively compare the results with relevant publications regarding the wild food plants of Belarus. We presume that wild plants are widely used in the region and remarkable overlap exists within the species used in both the food and medicinal areas.

\section{Data and methods}

\section{Wild plants}

In this article the concept of wild plants encompasses native and naturalized species not cultivated for food. The core of the selection is based on the internationally agreed upon ethnobotanical perception and refers primarily to plants growing without deliberate cultivation or those able to reproduce without human intervention 
[40-43]. It includes greenery trees (like Syringa vulgaris, Aesculus hippocastanum), but we excluded from the scope of this study all fruiting trees and shrubs cultivated for food purposes, even if they either run wild (like Prunus cerasus L. or Ribes rubrum L.) or have wild relatives (such as Ribes nigrum L. or Malus spp.), as a vast majority of people claimed that they collect fruits and other parts from the cultivated ones. As an exception we did include a taxon that was once cultivated, but now perceived somewhat as a nuisance (Armoracia rusticana). Other species that are cultivated for food purposes but tend to run wild (like Anethum graveolens L. or Nigella sativa L.) were excluded from this study.

\section{Region}

Belarus is located in Central and Eastern Europe, bordered by Lithuania to the north-west, Latvia to the north, Russia to the northeast and the east, Ukraine to the south, and Poland to the west. Its territory is about 207.6 thousand sq. $\mathrm{km}$. The climate of Belarus is moderately continental, transitional between maritime and continental. The climatic conditions are caused by the position of the country in the temperate latitudes of the western part of the East European Plain [44]. Belarus, more than other countries, was affected by the Chernobyl disaster, which caused the relocation of millions of people.

The whole territory of Belarus is part of the forest zone of Eastern Europe. Mixed forest is typical for Belarus, and from the north to the south there is a transition from boreal to nemoral forest types. Today, forests cover $37.8 \%$ of the total area, marshes $11.5 \%$, grasslands $15.8 \%$ and bushes $1.9 \%$. The vegetation varies with climate, soil type and topographic relief. Indigenous plants include about 100 species of trees and bushes as well as more than 1500 herbaceous plants [45]. The most common trees are conifers (pine, fir) and shrubs (juniper). The main deciduous species are birch, aspen, alder, as well as oaks and limes, and more rarely maple, hornbeam, ash, and poplar, among others. The most prominent shrubs are hazel, rowan, viburnum, buckthorn, raspberry, willow, vines, etc. The forests are also rich in mushrooms and berries, which includes strawberries, blueberries and cranberries in dry coniferous and mixed forests, and cranberries in swampy areas. Widespread meadow grasses include timothy, bluegrass, and various types of clover [45].

Liuban district is located in the southeast of the Minsk Region. The town of Liuban is the centre of the district which includes the township of Urečča and 125 rural settlements. The northern part of the district is located on the Central Biarezina plain, while the southern part is within the Prypiać Paliessie. In the central part of the district the Aresa River (a left-bank tributary of the
Prypiać) flows from north to south. Most areas of the Paliessie region have been drained. About 33\% of the area is covered by forests (coniferous and mixed deciduous forest, as well as birch, oak and alder that also grow there). The area is mostly agricultural and specializes in meat and milk cattle breeding, pig breeding and potato cultivation.

Interviews were conducted in eleven villages, which are part of the village council of Asaviec, Liubań district (Fig. 1); for more information on the district see [46]. About 2450 inhabitants live in the 15 villages of the village council of Asaviec. The working-age population is dominated by men (men: 770; women: 533), while the reverse is true for the senior population (590 women and 221 men). Bigger villages, in particular, are home to numerous migrants from both Belarussian and Ukrainian parts of the Chernobyl radiation zone. In recent years, migrants from Donbas have also become common. They were first warmly received, but later locals became disappointed with their attitude: locals perceive that migrants consider life in the Asaviec region too hard and this makes them unsatisfied.

Orthodox Christianity is the religion of the vast majority of the rural population, although the Catholicism is also practiced. Unemployment officially does not exist; all working-age people are employed or have official exemptions for not working. Yet, some people admit that younger men temporally work in towns or in Russia to sustain their families, which is probably one reason for encountering so few young men. Retail shops are present in seven larger villages, where 100 to 900 people live; inhabitants of smaller villages are supplied by mobile shops visiting twice a week. Jaminsk has a hospital, and Plastok has a medical aid station served by a medical assistant/midwife. While some bigger villages have a better-developed water system, small villages have surface water wells, which can dry out in summer, and often one well is shared by several households. Toilets are mainly outside and the dominant washing place is the sauna. Houses are traditionally decorated inside and outside; as the wealth of the villagers allow, some renovation work (new windows, painting) is done, supported mainly by relatives living in towns.

Many villagers still keep domestic animals (pigs, goats, ducks, hens, cows and horses), but admit that their numbers have diminished during the last several years. Smaller villages are built mainly in a linear fashion, so that houses are situated on either side of the road with few cross streets, making the settlement long but compact. Almost every household has a bench in front, outside the fence and along the street, so that home owners can "socialize" with fellow villagers sitting on these benches. A lot of effort is put into maintaining vegetable gardens: the selection of fresh vegetables in shops is quite modest, and so all greens and vegetables are grown 


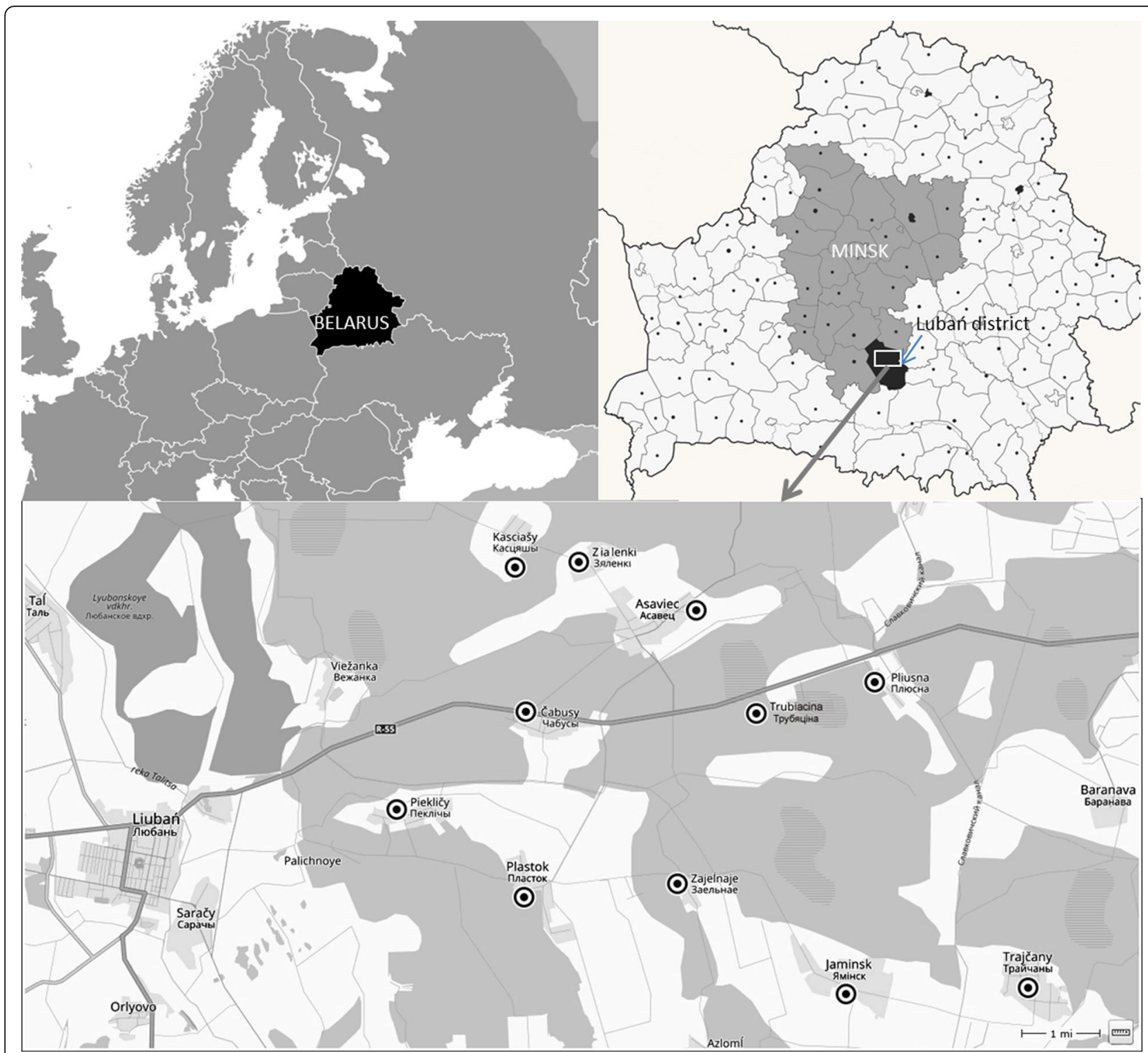

Fig. 1 Region under investigation

in relatively small garden plots close to houses. Flower gardens, which are considered the pride and joy of any good lady of the house, are more common in bigger villages, but even here they are confined to only a small part of the whole allotment.

\section{Data collection}

The collection of data on the use of wild plants was part of a wider ethnobotanical and ethnomedicinal field study, conducted in the Liubań district in May 2016 as a practical part of a development cooperation project financed by the Estonian Foreign Ministry. Interviewees were approached on a random basis, in an attempt to get a cross-section of the local population. Yet, this was not simple, as mainly only retired people were available for discussions during workdays, whereas other adults were working or busy with household tasks (on the weekend). The majority of people in the study sample were collective farm workers, and only few of them had secondary or higher education (working as officials or in the sphere of culture). For the analysis we selected responses of all 134 people who were borneither in the villages where the interviews took place or elsewhere in the district of Li ubań (mainly from neighbouring villages belonging to the same village council) and now living in the village in which they were interviewed. Of these respondents, 87 (64.9\%) were women and 47 (35.1\%) were men. The fewer number of men is due to low representation of elderly men in the villages. The mean age of the interviewees was 63.3 years; the oldest was 92 years old and the youngest was 27 years old. 
Interviews lasted from 0.5 to $2.5 \mathrm{~h}$ and were conducted either on the bench in front of the respondent's house or in their homes, in which researchers were often invited (and then fed or offered (ritual) drinks), especially when it was raining. The folk history method (reconstruction of historical events through the memory of common people, sensu [47]) was employed: in addition to documenting current uses, we also asked interviewees to recall the uses of plants they had used themselves or observed their parents using in the past, especially during their childhood. In the semi-structured interviews we approached the subject through uses, not through specific plants. First, we inquired about the use of wild plants for food, naming specific food-use categories (soups, other hot dishes, salads, jams, desserts, put in bread, used for seasoning food and drinks, and snacks). As the interview proceeded, we asked about using plants and other means in healing (headache, cold, sore throat, heart problems, stomach ache, foot ache, wounds, etc.) both people and animals (when animals get ill) and also for other purposes related to health and wellbeing (prophylactics, being healthy, care of skin and hair, disinfection, good scent, etc.). We let the respondents elaborate on the subject. Where weather and the interviewee's health or time permitted, walks in the gardens and surrounding meadows and forests were undertaken and voucher specimens collected. We also collected samples of dried plants available from the interviewees' homes. Although it was late spring and most of the stores were already used up, we encountered a high variety of stored plants in many households. Interviews were voicerecorded upon permission of the interviewee, and field notes were also taken. The purpose of the study was explained to every person and prior informed consent was obtained from all interviewees. We followed the Code of Ethics of the International Society of Ethnobiology [48]. All interviews were subsequently transcribed. The voicerecorded interviews as well as their transcripts are stored at the The Center for Belarusian Culture, Language and Literature Research within the Archives of The Institute of Art, Ethnography and Folklore, named after $\mathrm{K}$. Krapiva (AIAEF 23-16-2). Anonymized copies of interview transcripts are also stored at the Scientific Archive of the Estonian Folklore Institute (EFISA Valgevene2016) located in the Estonian Literary Museum.

Whenever possible plant voucher specimens were taken or plants identified on the basis of dried samples. As it was not yet the full vegetation season, some plants were identified on the basis of their vernacular name and a full description provided by the interviewee. In those cases when interviewees were treating a genus as one unit, the plant was identified at the genus level only, even if voucher specimens for some representatives of the genus were collected (for example Hypericum, Rosa,
Crataegus). This practice was followed as there is no guarantee that interviewees, at some point in their lives, did not collect representatives of other species belonging to the same genus.

Collected voucher specimens were dried and identified with the help of Toomas Kukk (Curator of the Estonian University of Life Sciences herbaria); vouchers are deposited at the Estonian University of Life Sciences herbaria (TAA), assigned herbarium numbers within the range TAA0132555-0132710, and also bearing numbers LJUB001-152. Dried plant samples collected from respondents are deposited at the Scientific Archive of the Estonian Folklore Institute (EFISA Valgevene2016, bearing numbers LJUD00185). Taxonomic identification, botanical nomenclature, and family assignments followed the Flora Europaea [49], The Plant List database [50], and the Angiosperm Phylogeny Group IV [51].

\section{Data analysis}

Interview records were digitalized and entered into a Microsoft Excel spread sheet. To follow emic categories, information was structured in Detailed Use Records (DUR adopted from [52]), where interviewees $(i)$ mention a specific use $(u$, e.g. emic disease category [cough, sore throat, heart disease, back pain, etc.], food category [snack, drink, condiment, soup, jam, etc.], emic veterinary treatment) of a plant part ( $p$, e.g. fruits, leaves, aerial parts, flowers, etc.) prepared in a certain way ( $w$, e.g. topical application of fresh plant, tea (plants macerated in hot water), decoction (plants boiled in water), tincture (plants macerated in alcohol-either applied or drunk), special preparation, etc.). Interviewee-defined emic categories were employed to ascertain local perceptions.

For every taxon, the number of Use Citations (UCnumber of people who claimed the (specific) use of the plant during the interview) and the number of DUR were calculated for the sum of all uses and separately for food, medicinal and veterinary areas. UC were also calculated for general and emic categories.

Following the recommendation given in several recent publications $[53,54]$ and to illustrate the diversity of various uses, uses mentioned by only one person were also included. Informant Consensus Factor (FIC [55]) was calculated for every use area as well as for different use categories within each of the three areas. The FIC is calculated as follows: number of UC minus the number of species used, divided by the number of use citations minus one.

Finally, the reliability criterion [56] was also assessed. The influence of age of the interviewees on the number of used plants and DURs was assessed by calculating $\mathrm{R}^{2}$ in Microsoft Excel. 


\section{Use areas}

For the comparative analysis, all DURs were attributed to one of three use areas (hereafter areas):

Food area-indicates that the use was related to food consumption, including hot and cold meals, drinks (including alcoholic ones), fermented foods, condiments, occasional snacks, the making of recreational teas (e.g. herbal beverages prepared as infusions or decoctions and consumed in a food context without folk medical indications sensu [57]), food preservatives and food preparation accessories (e.g. tree leaves put under bread during baking). Medicinal area-contains all uses related to the treatment or prevention of all diseases and illnesses that are locally known or diagnosed by a doctor, but also uses related to the perceived healthiness of a plant or product made from a plant, beauty procedures, insect repellent and the creation of a good atmosphere (contributing to wellbeing).

Veterinary area-covers all uses of wild plants related to the treatment of home animals or specially emphasized fodder, distinct from the general "hay, grains and potato" fodder.

For uses in the food area, qualitative comparison was made with data collected throughout Poland in 20th21st centuries [10], while for the other two areas such a comparison was not possible due to the lack of recent data.

\section{Results}

\section{Summary of the use of plants}

Altogether, 88 wild plant taxa belonging to 45 plant families were used across all three areas (Table 1). Sixtyseven $(76.1 \%)$ taxa met the reliability criterion, signifying that they were used by at least three people. We registered 2252 DUR of wild plants in total, with the food area dominating the variety of uses (54\%, 1216 DUR), followed by uses related to the medicinal area (43\%, 968 DUR) and the veterinary medicine and fodder area (3\%, 68 DUR).

The four plant families with the highest number of taxa and DUR were Asteraceae (12 taxa/206 DUR), Rosaceae (10 taxa/420 DUR), Ericaceae (8 taxa/501 DUR), and Betulaceae (3 taxa/170 DUR). Among the ten most diversely used taxa, the vast majority were fruit bearing shrubs (four Vaccinium genera and Rubus idaeus), trees (Betula spp., Tilia cordata and Quercus robur) and herbaceous taxa (Rumex spp. and Urtica dioica).

The two most knowledgeable interviewees provided 39 and 36 taxa and 73 and 81 uses, respectively. The two least knowledgeable respondents provided only one use.
The mean number of named taxa was 11.6 and mean number of DUR was 16.8. Although visually a small group of middle-aged women stand out as having more diverse knowledge, there was no statistically significant difference in the influence of age or sex of the respondents on either the number of used plants or the diversity of uses (Fig. 2).

\section{Food area}

In total, 58 taxa belonging to 31 families were used for food. Of these, only 19 taxa were mentioned by more than $10 \%$ of the interviewees, while the uses of 26 taxa were mentioned by less than three people; the reliability criterion was met by $55.2 \%$ (32) of the taxa. The most represented families were Rosaceae (10 taxa), Asteraceae (6 taxa) and Ericaceae (6 taxa). The families with the most varied food uses were Ericaceae (391 DUR), Rosaceae (240 DUR), Betulaceae (95 DUR), Polygonaceae (91 DUR), and Urticaceae (61 DUR). The most diversely used food taxa overlap greatly with the top taxa overall and only Fragaria vesca and Chenopodium album replaced Quercus robur and Tilia cordata, which dominate the list of medicinal plants.

Among the taxa used by at least three respondents, four were used solely for food : Chenopodium album, Oxalis acetosella, Carum carvi and Corylus avellana, but only the first of these was used by a large number of interviewees (41).

Among the 124 respondents who claimed to have used wild plants for food, the two most knowledgeable used 18 taxa, numbering 30 and 29 DUR respectively. However, there were seven people who mentioned only one use of wild plants for food. The mean number of used taxa was 7.3 and the mean number of DUR was 9.08.

All the DUR in the food area were distributed between 52 emic food categories, which can be attributed to 23 general food categories (Table 2). However, more than three-quarters of all DUR were distributed between six general food categories (Fig. 3).

The Informant Consensus Factor for the whole food area was 0.94 (910 use citations for 58 taxa). Only one general food category had a relatively high FIC while having only two utilized taxa: fresh and processed drinks $(\mathrm{FIC}=0.98)$, represented by tree sap. The category of kvass ( $\mathrm{FIC}=0.95$ ), which is also mainly tree sap based, contains three taxa; the same number of taxa was in the category of leaves that are put under bread (FIC = 0.88). Some of the dominate categories with a relatively wide variety of used taxa attained high FIC values; however, the majority of those contain some highly dominate taxa and about twice the number of taxa used by only a few people. Foremost in this group was the soup category $($ FIC $=0.96)$, where among seven taxa three were highly prevalent (Rumex acetosa, Chenopodium album 
Table 1 Wild plants used in food, medicinal and veterinary areas

\begin{tabular}{|c|c|c|c|c|c|}
\hline Family, taxa, voucher no. & Local names & Used parts & Mode of use & $\begin{array}{l}\text { Emic use } \\
\text { category }\end{array}$ & $U C$ \\
\hline \multicolumn{6}{|l|}{ Acoraceae } \\
\hline \multirow[t]{3}{*}{ Acorus calamus L. } & \multirow[t]{3}{*}{ aip, плюшнік/air, pliušnik } & Aerial parts & Placed in water & Disinfectant & 1 \\
\hline & & Roots & Ritual wearing & Evil eye & 1 \\
\hline & & $\begin{array}{l}\text { Stem (lower, } \\
\text { white part) }\end{array}$ & Fresh & Snack & 1 \\
\hline \multicolumn{6}{|l|}{ Adoxaceae } \\
\hline \multirow[t]{30}{*}{ Viburnum opulus L., (LUBB036) } & \multirow[t]{30}{*}{ каліна/kalina } & \multirow[t]{28}{*}{ Fruits } & Dried & Recreational tea & 1 \\
\hline & & & Dried on twigs & Snack & 1 \\
\hline & & & \multirow[t]{2}{*}{ Fermented } & Heart diseases & 2 \\
\hline & & & & Hypertension & 2 \\
\hline & & & \multirow[t]{13}{*}{ Fresh } & Cold & 2 \\
\hline & & & & Compote & 1 \\
\hline & & & & Dessert & 1 \\
\hline & & & & Fruit water & 2 \\
\hline & & & & Heart problems & 1 \\
\hline & & & & Hypertension & 1 \\
\hline & & & & Jam & 5 \\
\hline & & & & Kissel & 1 \\
\hline & & & & $\begin{array}{l}\text { Taste additive to } \\
\text { strong alcohol }\end{array}$ & 6 \\
\hline & & & & Snack & 1 \\
\hline & & & & Sore throat & 1 \\
\hline & & & & Syrup & 1 \\
\hline & & & & Wine & 1 \\
\hline & & & \multirow[t]{2}{*}{ Frozen } & Snack & 1 \\
\hline & & & & Raw jam & 1 \\
\hline & & & In sugar & Preserve & 1 \\
\hline & & & Jam & Cold & 1 \\
\hline & & & \multirow[t]{2}{*}{ Raw jam } & Cough & 2 \\
\hline & & & & Heart problems & 1 \\
\hline & & & \multirow[t]{4}{*}{ Tincture } & Healthy & 1 \\
\hline & & & & Heart problems & 1 \\
\hline & & & & Hypertension & 4 \\
\hline & & & & Stomach ache & 1 \\
\hline & & & Tea & Cough & 1 \\
\hline & & Flowers & Tea & Hypertension & 1 \\
\hline & & Leaves & Decoction & Sore throat & 1 \\
\hline \multicolumn{6}{|l|}{ Amaranthaceae } \\
\hline Atriplex hortensis L. & лебяда красная/liebiada krasnaja & Aerial parts & Fresh & Soup & 2 \\
\hline \multirow[t]{3}{*}{ Chenopodium album L., (LUB017) } & \multirow[t]{3}{*}{ лебяда, лябяда/liebiada, liabiada } & \multirow[t]{3}{*}{ Aerial parts } & \multirow[t]{3}{*}{ Fresh } & Salad & 3 \\
\hline & & & & Snack & 3 \\
\hline & & & & Soup & 35 \\
\hline
\end{tabular}

Amaryllidaceae

Allium ursinum L., (LUB002) 
Table 1 Wild plants used in food, medicinal and veterinary areas (Continued)

\begin{tabular}{|c|c|c|c|c|c|}
\hline & $\begin{array}{l}\text { дзікі часнок, чарамша, медвежы } \\
\text { лук/dziki časnok, čaramša, }\end{array}$ & & & $\begin{array}{l}\text { Condiment for } \\
\text { meat }\end{array}$ & \\
\hline & & & Fresh & Salad & 11 \\
\hline & & & & Snack & 5 \\
\hline & & & & Soup & 2 \\
\hline & & & & Condiment & 3 \\
\hline & & & & $\begin{array}{l}\text { Condiment for } \\
\text { soup }\end{array}$ & 1 \\
\hline & & & & Vitamins & 1 \\
\hline & & & Frozen & Snack & 1 \\
\hline & & & Marinated & Condiment & 2 \\
\hline & & Roots & Fresh & Snack & 1 \\
\hline Apiaceae & & & & & \\
\hline $\begin{array}{l}\text { Aegopodium podagraria L., } \\
\text { (LJUD035) }\end{array}$ & сныць/snyć & Leaves & Fresh & Salad & 1 \\
\hline Carum carvi L., (LUUB132) & кмін, тмін/kmin, tmin & Seeds & Dried & Recreational tea & 1 \\
\hline & & & & Condiment & 3 \\
\hline & & & & $\begin{array}{l}\text { Condiment for } \\
\text { bread }\end{array}$ & 4 \\
\hline & & & & $\begin{array}{l}\text { Condiment for } \\
\text { cheese }\end{array}$ & 1 \\
\hline & & & & $\begin{array}{l}\text { Condiment for } \\
\text { meat }\end{array}$ & 1 \\
\hline & & & & $\begin{array}{l}\text { Condiment for } \\
\text { sausages }\end{array}$ & 1 \\
\hline Asparagaceae & & & & & \\
\hline Convallaria majalis $\mathrm{L}$. & ландыш/landyš & Flowers & Tincture & Heart disease & 2 \\
\hline $\begin{array}{l}\text { Maianthemum bifolium (L.) } \\
\text { F.W.Schmidt, (LJUB065) }\end{array}$ & майнік/majnik & Fruits & Fresh & Snack & 1 \\
\hline Asteraceae & & & & & \\
\hline Achillea millefolium L., (LUUB118) & падбел, тысячаліснік/padbiel, & Aerial parts & Decoction & Heart problems & 1 \\
\hline & & & Fresh & Fodder for turkey & 1 \\
\hline & & & Tea & Bile neutralizer & 1 \\
\hline & & & & Gastritis & 1 \\
\hline & & & & Stomach ache & 1 \\
\hline & & Inflorescences & Tea & Panacea & 1 \\
\hline & & & & Stomach ache & 2 \\
\hline & & & & Women diseases & 1 \\
\hline & & Leaves & Decoction & Women diseases & 1 \\
\hline & & & Topical application & Cuts & 2 \\
\hline & & & & Wounds & 2 \\
\hline Arctium tomentosum Mill., (LJUB019) & лапух, лопух, ваўчкі, дзяды, & Inflorescences & Decoction & Hair care & 2 \\
\hline & $\begin{array}{l}\text { Дзядоуунік, рапейнік/lapuch, } \\
\text { lopuch, vaǔčki, dziady, } \\
\text { dziadoŭnik, rapieinik }\end{array}$ & Leaves & Fresh & $\begin{array}{l}\text { Put under bread } \\
\text { when baked }\end{array}$ & 1 \\
\hline & & & Topical application & Back pain & 1 \\
\hline & & & & Bruises & 1 \\
\hline & & & & Foot ache & 3 \\
\hline & & & & Joint pain & 9 \\
\hline
\end{tabular}


Table 1 Wild plants used in food, medicinal and veterinary areas (Continued)

\begin{tabular}{|c|c|c|c|c|c|}
\hline & & & & Knee ache & 2 \\
\hline & & & & Pain & 1 \\
\hline & & & & Painful place & 3 \\
\hline & & & & Rheumatic pains & 2 \\
\hline & & & & Tumour & 1 \\
\hline & & & & Wounds & 1 \\
\hline & & Roots & Decoction & Hair care & 3 \\
\hline & & & & Joint pain & 1 \\
\hline & & & Fresh & Vitamins & 1 \\
\hline & & & Fresh, greasing & Joint pain & 4 \\
\hline & & & Tincture, topical & Foot ache & 1 \\
\hline \multirow[t]{2}{*}{ Arnica montana L. } & \multirow[t]{2}{*}{ арніка горная/arnika hornaja } & Whole plant & Tea & Heart problems & 1 \\
\hline & & & & Nerves & 1 \\
\hline \multirow[t]{13}{*}{ Artemisia absinthium L., (LUB001) } & \multirow[t]{13}{*}{$\begin{array}{l}\text { палын (серы), палыннік/palyn } \\
\text { (siery), palynnik }\end{array}$} & Aerial parts & Decoction & $\begin{array}{l}\text { Appetizer for } \\
\text { cows }\end{array}$ & 1 \\
\hline & & & & $\begin{array}{l}\text { Diarrhoea in } \\
\text { chicken }\end{array}$ & 1 \\
\hline & & & & Diarrhoea in cows & 3 \\
\hline & & & & Diarrhoea in pigs & 5 \\
\hline & & & & $\begin{array}{l}\text { Rumination } \\
\text { problems in cows }\end{array}$ & 3 \\
\hline & & & Dried & Fodder for rabbits & 2 \\
\hline & & & Fresh & $\begin{array}{l}\text { Disinfectant for } \\
\text { home animals }\end{array}$ & 1 \\
\hline & & & & Fodder for turkey & 1 \\
\hline & & & $\begin{array}{l}\text { Tincture, topical } \\
\text { application }\end{array}$ & Joint pain & 1 \\
\hline & & & Covered with & $\begin{array}{l}\text { Preservative for } \\
\text { potatoes }\end{array}$ & 1 \\
\hline & & & Tea & Appetizer & 2 \\
\hline & & & & Diarrhoea & 6 \\
\hline & & & & $\begin{array}{l}\text { Helminthic } \\
\text { infection }\end{array}$ & 1 \\
\hline \multirow[t]{4}{*}{ Artemisia vulgaris L., (LJUB139) } & \multirow{4}{*}{$\begin{array}{l}\text { чарнабы'льнік, чорны палын, } \\
\text { быльнік/с̌arnaby'Ínik, čorny } \\
\text { palyn, byÍnik }\end{array}$} & Aerial parts & Dried & $\begin{array}{l}\text { Blood in urine in } \\
\text { cows }\end{array}$ & 1 \\
\hline & & & & fodder for rabbits & 1 \\
\hline & & & Fresh & Recreational tea & 1 \\
\hline & & & Tea & Diarrhoea & 2 \\
\hline \multirow[t]{3}{*}{ Bidens tripartita $\mathrm{L}$. } & \multirow[t]{3}{*}{$\begin{array}{l}\text { ваўчкі, чарада, чэрада/vaŭс̌ki, } \\
\text { čarada, čerada }\end{array}$} & Aerial part & Bath & $\begin{array}{l}\text { Diathesis in } \\
\text { children }\end{array}$ & 6 \\
\hline & & & & Calming & 1 \\
\hline & & & & Skin diseases & 1 \\
\hline \multirow[t]{5}{*}{ Cyanus segetum Hill, (LJUD023) } & \multirow[t]{5}{*}{ васількі/vasiĺki } & Aerial parts & Tea & Kidney diseases & 1 \\
\hline & & Inflorescences & Bath & $\begin{array}{l}\text { Diathesis in } \\
\text { children }\end{array}$ & 1 \\
\hline & & & Compress & Eye problems & 1 \\
\hline & & & Dried & Recreational tea & 2 \\
\hline & & & Tea & Healthy & 1 \\
\hline
\end{tabular}


Table 1 Wild plants used in food, medicinal and veterinary areas (Continued)

\begin{tabular}{|c|c|c|c|c|c|}
\hline \multirow{7}{*}{$\begin{array}{l}\text { Helichrysum arenarium (L.) Moench, } \\
\text { (LJUD007) }\end{array}$} & \multirow{7}{*}{$\begin{array}{l}\text { бяссмертнік (пясчаны)/ } \\
\text { biassmiertnik (piasčany) }\end{array}$} & \multirow[t]{7}{*}{ Aerial parts } & Dried & Recreational tea & 1 \\
\hline & & & \multirow[t]{6}{*}{ Tea } & Bile deficiency & 1 \\
\hline & & & & Gall stones & 1 \\
\hline & & & & Healthy & 1 \\
\hline & & & & Liver diseases & 5 \\
\hline & & & & Panacea & 2 \\
\hline & & & & Stomach ache & 2 \\
\hline \multirow{13}{*}{$\begin{array}{l}\text { Matricaria spp. (Incl. Matricaria } \\
\text { chamomilla L., (LUUD025) and } \\
\text { Matricaria suaveolens Koch) }\end{array}$} & \multirow{13}{*}{$\begin{array}{l}\text { рамонак, рамашка/ramonak, } \\
\text { ramaška }\end{array}$} & \multirow[t]{5}{*}{ Aerial parts } & \multirow[t]{3}{*}{ Decoction } & Calming & 1 \\
\hline & & & & Hair care & 1 \\
\hline & & & & Wounds & 1 \\
\hline & & & \multirow[t]{2}{*}{ Tea } & Sore throat & 1 \\
\hline & & & & Stomach ache & 1 \\
\hline & & \multirow[t]{8}{*}{ Inflorescences } & Compress & Eye problems & 2 \\
\hline & & & \multirow[t]{6}{*}{ Decoction } & Dandruff & 1 \\
\hline & & & & Hair care & 1 \\
\hline & & & & Heart problems & 1 \\
\hline & & & & Haemorrhoids & 1 \\
\hline & & & & $\begin{array}{l}\text { Organism } \\
\text { cleansing }\end{array}$ & 2 \\
\hline & & & & Women diseases & 1 \\
\hline & & & Dried, eaten & Diarrhoea & 1 \\
\hline \multirow[t]{15}{*}{ Tanacetum vulgare L., (LJUB108) } & \multirow{15}{*}{$\begin{array}{l}\text { цытва'р, цытва'рная палынь, } \\
\text { піжма/сytva'r, cytva'rnaja palyń, } \\
\text { pižma }\end{array}$} & \multirow[t]{4}{*}{ Aerial parts } & Fresh & $\begin{array}{l}\text { Helminthic } \\
\text { infection }\end{array}$ & 1 \\
\hline & & & \multirow[t]{3}{*}{ Tea } & Diarrhoea & 2 \\
\hline & & & & $\begin{array}{l}\text { Helminthic } \\
\text { infection }\end{array}$ & 1 \\
\hline & & & & Stomach ache & 2 \\
\hline & & \multirow[t]{9}{*}{ Inflorescences } & Decoction & $\begin{array}{l}\text { Helminthic } \\
\text { infection }\end{array}$ & 2 \\
\hline & & & \multirow[t]{4}{*}{ Dried, added to fodder } & $\begin{array}{l}\text { Helminthic } \\
\text { infection in cows }\end{array}$ & 1 \\
\hline & & & & $\begin{array}{l}\text { Helminthic } \\
\text { infection in pigs }\end{array}$ & 1 \\
\hline & & & & $\begin{array}{l}\text { Strengthening of } \\
\text { cows }\end{array}$ & 1 \\
\hline & & & & $\begin{array}{l}\text { Strengthening of } \\
\text { pigs }\end{array}$ & 1 \\
\hline & & & \multirow[t]{2}{*}{ Eaten fresh } & $\begin{array}{l}\text { Helminthic } \\
\text { infection }\end{array}$ & 1 \\
\hline & & & & Stomach ache & 1 \\
\hline & & & \multirow[t]{2}{*}{ Tea } & Diarrhoea & 1 \\
\hline & & & & $\begin{array}{l}\text { Helminthic } \\
\text { infection }\end{array}$ & 1 \\
\hline & & \multirow[t]{2}{*}{ Leaves } & \multirow[t]{2}{*}{ Tea } & Diarrhoea & 1 \\
\hline & & & & $\begin{array}{l}\text { Helminthic } \\
\text { infection }\end{array}$ & 1 \\
\hline \multirow{2}{*}{$\begin{array}{l}\text { Taraxacum officinale (L.) Weber ex } \\
\text { F.H.Wigg., (LUUB122) }\end{array}$} & \multirow{2}{*}{$\begin{array}{l}\text { дзьмухавец, адуванчыкі/ } \\
\text { dźmuchaviec, aduvančyki }\end{array}$} & \multirow[t]{2}{*}{ Aerial parts } & Fresh & Fodder for pigs & 1 \\
\hline & & & $\begin{array}{l}\text { Tincture, topical } \\
\text { application }\end{array}$ & Joint pain & 1 \\
\hline
\end{tabular}


Table 1 Wild plants used in food, medicinal and veterinary areas (Continued)

\begin{tabular}{|c|c|c|c|c|c|}
\hline & & Inflorescences & Decoction & Joint pain & 1 \\
\hline & & & & Kidney diseases & 1 \\
\hline & & & Fermented with sugar & Immune boosting & 1 \\
\hline & & & Fresh & Cold & 1 \\
\hline & & & & Fodder for pigs & 1 \\
\hline & & & & Good for cows & 1 \\
\hline & & & & Good for horses & 1 \\
\hline & & & & Jam & 10 \\
\hline & & & Fresh, topical application & Joint pain & 2 \\
\hline & & & Jam & Bronchitis & 1 \\
\hline & & & & Cancer & 1 \\
\hline & & & & Cold & 1 \\
\hline & & & & Sore throat & 1 \\
\hline & & & & Tuberculosis & 1 \\
\hline & & & Tincture & Stomach ache & 1 \\
\hline & & & $\begin{array}{l}\text { Tincture, topical } \\
\text { application }\end{array}$ & Joint pain & 1 \\
\hline & & & Syrup & Vitamins & 1 \\
\hline & & Leaves & Decoction & Hair care & 1 \\
\hline & & & Fresh & Fodder for pigs & 1 \\
\hline & & & & $\begin{array}{l}\text { Fodder for home } \\
\text { animals }\end{array}$ & 1 \\
\hline & & & & Salad & 7 \\
\hline & & & Tincture & Stomach ache & 1 \\
\hline & & Roots & Coffee substitute & Kidney stones & 1 \\
\hline & & & Dried & Coffee substitute & 1 \\
\hline & & & & Recreational tea & 1 \\
\hline & & & Fresh, eaten & Cancer & 1 \\
\hline Tussilago farfara L., (LJUB023) & $\begin{array}{l}\text { падбел, маці-мачыха, маць-мачыха/ } \\
\text { padbiel, maci-mačycha, mać-mačycha }\end{array}$ & Aerial parts & Topical application & $\begin{array}{l}\text { Inflammation } \\
\text { processes }\end{array}$ & 1 \\
\hline & & Inflorescences & Tea & Cold & 1 \\
\hline & & & & Panacea & 1 \\
\hline & & Leaves & Decoction & Healthy & 1 \\
\hline & & & & Headache & 1 \\
\hline & & & & Sore throat & 1 \\
\hline & & & Tea & Cold & 1 \\
\hline & & & & Cough & 5 \\
\hline & & & & Expectorant & 1 \\
\hline & & & & Panacea & 1 \\
\hline & & & Topical application & Headache & 1 \\
\hline & & & & Wounds & 1 \\
\hline Betulaceae & & & & & \\
\hline Alnus spp. & альха, алешнік/aĺcha, aliešnik & Buds & Bath & Foot hatching & 1 \\
\hline & & Cone-like & Decoction & Diarrhoea in cows & 1 \\
\hline & & & & Diarrhoea in pigs & 1 \\
\hline & & & & Diarrhoea & 4 \\
\hline
\end{tabular}


Table 1 Wild plants used in food, medicinal and veterinary areas (Continued)

\begin{tabular}{|c|c|c|c|c|c|}
\hline & & & & Stomach ache & 1 \\
\hline \multirow[t]{41}{*}{$\begin{array}{l}\text { Betula spp., (incl. Betula pendula } \\
\text { Roth (LJUB081)) }\end{array}$} & $\begin{array}{l}\text { бяроза, } \\
\text { берёеза/biaroza, berioza }\end{array}$ & Buds & Dried & $\begin{array}{l}\text { Taste additive to } \\
\text { strong alcohol }\end{array}$ & 1 \\
\hline & & & Tincture & Diarrhoea & 2 \\
\hline & & & & Epilepsy & 2 \\
\hline & & & & Gastric ulcer & 1 \\
\hline & & & & Gastritis & 1 \\
\hline & & & & Healthy & 1 \\
\hline & & & & Kidney diseases & 3 \\
\hline & & & & Panacea & 3 \\
\hline & & & & Sore throat & 2 \\
\hline & & & & Stomach ache & 3 \\
\hline & & & & $\begin{array}{l}\text { Strengthening of } \\
\text { organism }\end{array}$ & 1 \\
\hline & & & & Wounds & 3 \\
\hline & & & Ritually fermented, bath & Epilepsy & 1 \\
\hline & & & Tea & Cold & 2 \\
\hline & & & & Diuretic & 1 \\
\hline & & & & Heart problems & 1 \\
\hline & & & & Panacea & 2 \\
\hline & & & & Sore throat & 1 \\
\hline & & Catkins & Tincture & Diarrhoea & 1 \\
\hline & & & & Gastric ulcer & 1 \\
\hline & & & & Gastritis & 1 \\
\hline & & & & Panacea & 1 \\
\hline & & & & Stomach ache & 1 \\
\hline & & & $\begin{array}{l}\text { Tincture, topical } \\
\text { application }\end{array}$ & Joint pain & 1 \\
\hline & & & Macerated in water & $\begin{array}{l}\text { Diarrhoea in } \\
\text { calves }\end{array}$ & 1 \\
\hline & & & Tea & Cold & 1 \\
\hline & & Leaves & Bath & Allergy & 1 \\
\hline & & & Dried & Recreational tea & 2 \\
\hline & & & Tea & Cold & 1 \\
\hline & & & & Heart problems & 1 \\
\hline & & & & Panacea & 1 \\
\hline & & & & Stomach ache & 1 \\
\hline & & & Topical application & Foot ache & 1 \\
\hline & & Resin & Topical application & Wounds & 2 \\
\hline & & Sap & Fermented & Kvass & 36 \\
\hline & & & Fresh & Drink & 30 \\
\hline & & & & Instead of water & 2 \\
\hline & & & & Diuretic & 1 \\
\hline & & & & $\begin{array}{l}\text { Increase volume } \\
\text { of breast milk }\end{array}$ & 1 \\
\hline & & & Processed & Drink & 16 \\
\hline & & & & Kidney diseases & 1 \\
\hline
\end{tabular}


Table 1 Wild plants used in food, medicinal and veterinary areas (Continued)

\begin{tabular}{|c|c|c|c|c|c|}
\hline & & Twigs & Bath & Prophylactics & 1 \\
\hline & & & Whisked in sauna & Back pain & 1 \\
\hline & & & & Healthy & 5 \\
\hline & & & & $\begin{array}{l}\text { Promotes } \\
\text { bloodstream }\end{array}$ & 1 \\
\hline & & & & Prophylactics & 10 \\
\hline Corylus avellana $\mathrm{L}$. & арэх (лясны), фундук, арэшына/ & Seeds & Dried & Snack & 3 \\
\hline & arech (liasny), tunduk, aresyna & & Fresh & Snack & 5 \\
\hline Boraginaceae & & & & & \\
\hline Pulmonaria spp. & медуніца/miedunica & Flowers & Fresh & Snack & 1 \\
\hline Symphytum officinale L. & акопнік/akopnik & Leaves & Topical application & Bruises & 1 \\
\hline & & Roots & Tincture & $\begin{array}{l}\text { Adhesion of } \\
\text { bones }\end{array}$ & 1 \\
\hline Brassicaceae & & & & & \\
\hline $\begin{array}{l}\text { Armoracia rusticana Gaertn. et al., } \\
\text { (LUUB093) }\end{array}$ & хрэн/chren & Leaves & Fresh & $\begin{array}{l}\text { Preservative for } \\
\text { preserves }\end{array}$ & 1 \\
\hline & & & & Condiment & 3 \\
\hline & & & & $\begin{array}{l}\text { Condiment for } \\
\text { lactofermented } \\
\text { cucumbers }\end{array}$ & 8 \\
\hline & & & & $\begin{array}{l}\text { Condiment for } \\
\text { preserves }\end{array}$ & 1 \\
\hline & & & Topical application & Back pain & 1 \\
\hline & & & & Joint pain & 2 \\
\hline & & Roots & Dried & $\begin{array}{l}\text { Condiment for } \\
\text { meat }\end{array}$ & 1 \\
\hline & & & Fresh & Appetizer & 1 \\
\hline & & & & Disinfectant & 1 \\
\hline & & & & Healthy & 1 \\
\hline & & & & $\begin{array}{l}\text { Taste additive to } \\
\text { strong alcohol }\end{array}$ & 1 \\
\hline & & & & Salad & 3 \\
\hline & & & & Snack & 2 \\
\hline & & & & Condiment & 13 \\
\hline & & & & $\begin{array}{l}\text { Condiment for } \\
\text { beet juice }\end{array}$ & 1 \\
\hline & & & & $\begin{array}{l}\text { Condiment for } \\
\text { lactofermented } \\
\text { cucumbers }\end{array}$ & 1 \\
\hline & & & & $\begin{array}{l}\text { Condiment for } \\
\text { meat }\end{array}$ & 3 \\
\hline & & & & $\begin{array}{l}\text { Condiment for } \\
\text { pork fat }\end{array}$ & 1 \\
\hline & & & Macerated in beer & Diabetes & 1 \\
\hline Capsella bursa-pastoris (L.) Medik., & пастуш'я сумка, сумачка, сушанка/ & Aerial parts & Decoction & Haemostatic & 1 \\
\hline & & & Tea & Diarrhoea & 1 \\
\hline & & & & Stomach ache & 1 \\
\hline & & & & Urinary bladder & 1 \\
\hline & & Seeds & Dried & Bread additive & 1 \\
\hline
\end{tabular}


Table 1 Wild plants used in food, medicinal and veterinary areas (Continued)

\begin{tabular}{|c|c|c|c|c|c|}
\hline \multicolumn{6}{|l|}{ Cannabaceae } \\
\hline \multirow[t]{5}{*}{ Humulus lupulus L., (LJUD027) } & \multirow[t]{5}{*}{ хмель/chmiel } & \multirow[t]{4}{*}{ Cones } & Decoction & Insomnia & 1 \\
\hline & & & Dried & Beer & 1 \\
\hline & & & \multirow[t]{2}{*}{ Fresh } & Bread additive & 1 \\
\hline & & & & $\begin{array}{l}\text { Taste additive to } \\
\text { strong alcohol }\end{array}$ & 1 \\
\hline & & Leaves & Dried & Beer & 1 \\
\hline \multicolumn{6}{|l|}{ Caprifoliaceae } \\
\hline \multirow[t]{12}{*}{ Valeriana officinalis $\mathrm{L}$. } & \multirow{12}{*}{$\begin{array}{l}\text { валерьян, валяр'янка/valieŕjan, } \\
\text { valiarjanka }\end{array}$} & \multirow[t]{5}{*}{ Aerial parts } & Dried & Recreational tea & 1 \\
\hline & & & Tincture & Healthy & 1 \\
\hline & & & \multirow[t]{3}{*}{ Tea } & Headache & 1 \\
\hline & & & & Heart problems & 2 \\
\hline & & & & Sedative & 1 \\
\hline & & \multirow[t]{7}{*}{ Roots } & \multirow[t]{3}{*}{ Decoction } & Heart problems & 2 \\
\hline & & & & Joint pain & 1 \\
\hline & & & & $\begin{array}{l}\text { Organism } \\
\text { cleansing }\end{array}$ & 1 \\
\hline & & & \multirow[t]{2}{*}{ Tincture } & Calming & 1 \\
\hline & & & & Heart problems & 2 \\
\hline & & & \multirow[t]{2}{*}{ Tea } & Calming & 1 \\
\hline & & & & Headache & 1 \\
\hline \multicolumn{6}{|l|}{ Caryophyllaceae } \\
\hline \multirow[t]{6}{*}{ Stellaria media (L.) Vill., (LJUB024) } & \multirow{6}{*}{$\begin{array}{l}\text { макрэц, макріца, звесчатка/makrec, } \\
\text { makrica, zviesčatka }\end{array}$} & \multirow[t]{6}{*}{ Aerial parts } & Decoction & Cancer & 1 \\
\hline & & & Fresh & Salad & 2 \\
\hline & & & $\begin{array}{l}\text { Tincture, topical } \\
\text { application }\end{array}$ & Rheumatic pains & 1 \\
\hline & & & \multirow[t]{3}{*}{ Fresh, topical application } & Foot sores & 1 \\
\hline & & & & Headache & 1 \\
\hline & & & & Rheumatic pains & 2 \\
\hline \multicolumn{6}{|l|}{ Cupressaceae } \\
\hline \multirow[t]{7}{*}{ Juniperus communis L. } & \multirow{7}{*}{$\begin{array}{l}\text { ядловец, мажжавельнік/jadloviec, } \\
\text { mažžavielnik }\end{array}$} & Galbules & Tincture & Back pain & 1 \\
\hline & & Leaves & Decoction & Bronchitis & 1 \\
\hline & & Pollen & Powder & Skin diseases & 1 \\
\hline & & \multirow[t]{4}{*}{ Twigs } & \multirow[t]{2}{*}{ Tea } & Cold & 1 \\
\hline & & & & $\begin{array}{l}\text { Cold in } \\
\text { domesticated } \\
\text { animals }\end{array}$ & 1 \\
\hline & & & \multirow[t]{2}{*}{ Whisked in sauna } & Healthy & 2 \\
\hline & & & & Prophylactics & 2 \\
\hline \multicolumn{6}{|l|}{ Dryopteridaceae } \\
\hline $\begin{array}{l}\text { Dryopteris carthusiana (Vill.) H.P. } \\
\text { Fuchs, (LUB083) }\end{array}$ & папаратнік/paparatnik & Leaves & Bath & Rejuvenating & 1 \\
\hline Equisetaceae & & & & & \\
\hline Equisetum arvense L., (LJUB120) & хвошч палявы/chvošč paliavy & Aerial parts & Decoction & $\begin{array}{l}\text { Bacterial diseases } \\
\text { in bees }\end{array}$ & 1 \\
\hline & & & Tea & Diuretic & 1 \\
\hline & & & & Healthy & 1 \\
\hline
\end{tabular}


Table 1 Wild plants used in food, medicinal and veterinary areas (Continued)

Kidney diseases

Ericaceae

Arctostaphylos uva-ursi (L.) Spreng.
Calluna vulgaris (L.) Hull, (LUDD079)
Chimaphila umbellata (L.) Nutt.,
(LUB146)

Ledum palustre L., (LUD052)

\section{талакнянка/talaknianka \\ Bepeck/vieriesk \\ станаўнік/stanaŭnik}

багон, багоўнік, буячнік/bahon, bahoŭnik, bujačnik

$\begin{array}{ll}\text { Aerial parts } & \text { Tea } \\ \text { Flowers } & \text { Dried } \\ \text { Aerial parts } & \text { Tea } \\ & \text { Tincture } \\ \text { Aerial parts } & \text { Decoction }\end{array}$

чарніка, чарнічнік, іванавыя ягады, чорныя ягады, ягоднік, ягады (лесные)/čarnicy, čarnika, čarničnik, ivanavyja jahady, čornyja jahady, jahodnik, jahady (liesnyje)

$\begin{array}{ll} & \\ & \\ & \text { Dried } \\ & \text { Smoked } \\ & \text { Tea } \\ & \\ \text { Aerial parts } & \text { Dried } \\ & \text { Tincture } \\ & \text { Tea } \\ & \\ & \text { Topical application } \\ & \text { Compote }\end{array}$

Decoction

Dried

Dried, ritual

Fresh
Kidney diseases 1

Recreational tea 1

Stomach ache 1

Stomach ache 1

Diarrhoea in 1

calves

Diarrhoea in cows 1

Bronchitis 1

Diarrhoea 1

Recreational tea 1

Tick prevention 1

Cough 3

Nerves

Recreational tea 6

Improve vision 1

Diabetes 1

Panacea 1

Rejuvenating 1

Eye problems 1

Improve vision 1

Cold 1

Compote 8

Constipation 1

Diabetes 1

Diarrhoea 12

Eye problems 4

Healthy 1

Taste additive to 1 strong alcohol

Recreational tea 4

Snack 8

Stomach ache 7

Teething pain in 1

children

Dessert 1

Stomach ache 1

Compote $\quad 26$

Dessert 3

Diarrhoea 2

Eye problems 5

Fruit water 1

Healthy 1

Hypotension 1

Improve vision 3 
Table 1 Wild plants used in food, medicinal and veterinary areas (Continued)

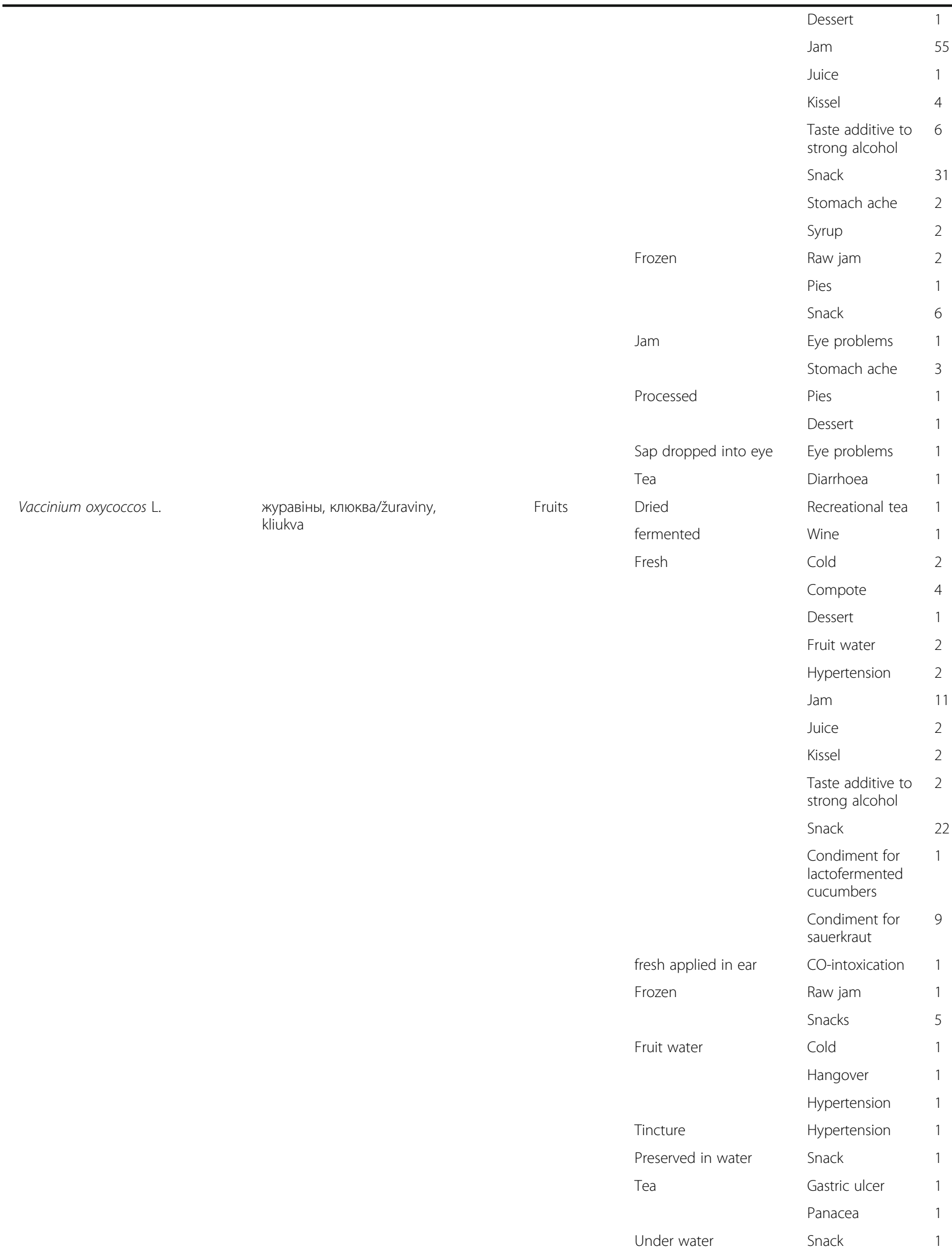


Table 1 Wild plants used in food, medicinal and veterinary areas (Continued)

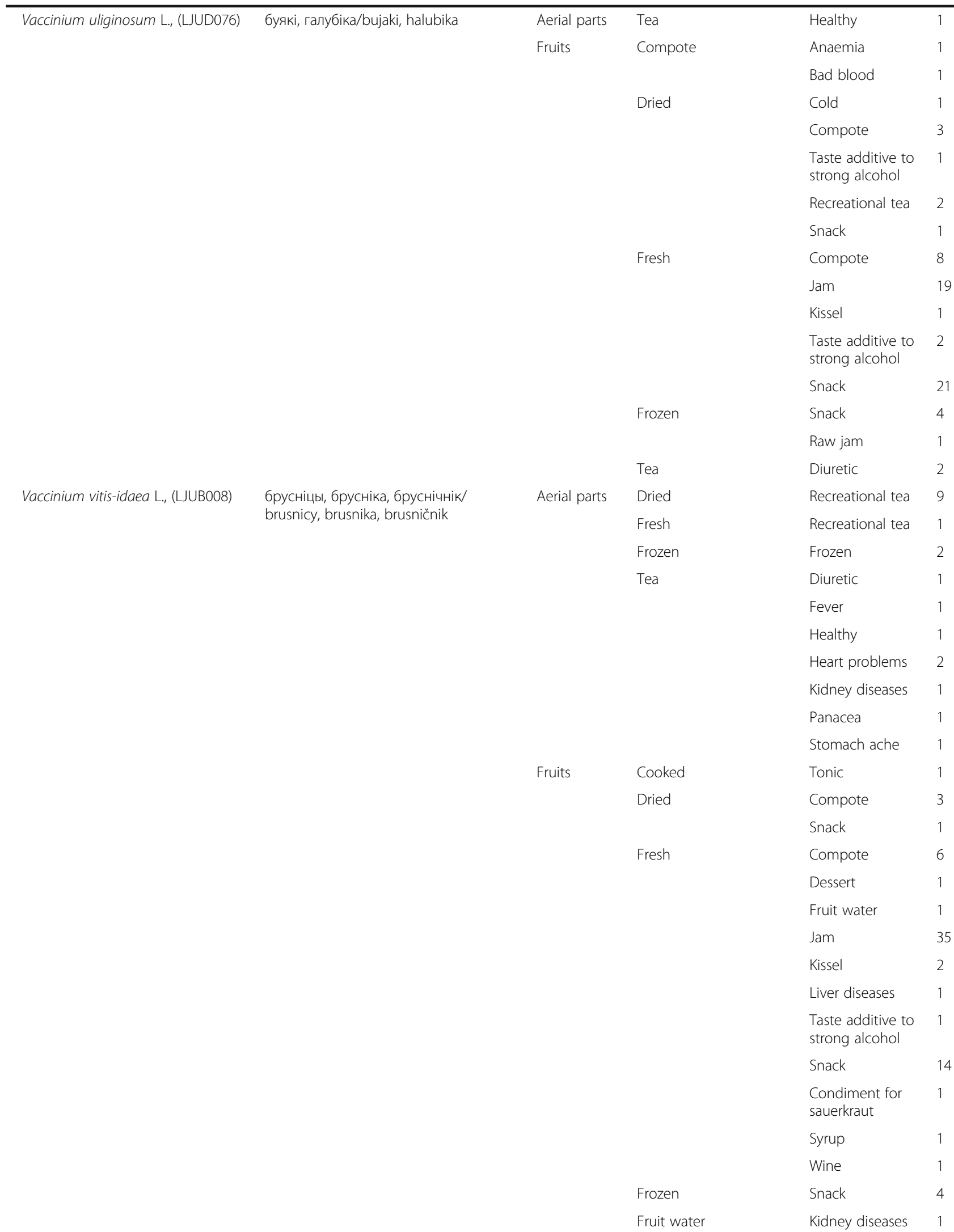


Table 1 Wild plants used in food, medicinal and veterinary areas (Continued)

\begin{tabular}{|c|c|c|c|c|c|}
\hline & & & & Sore throat & 1 \\
\hline & & & Processed & Pies & 2 \\
\hline & & & Refrigerated & Raw jam & 1 \\
\hline & & & Tea & Healthy & 1 \\
\hline & & & & $\begin{array}{l}\text { Organism } \\
\text { cleaning }\end{array}$ & 1 \\
\hline & & Leaves & Dried & Recreational tea & 5 \\
\hline & & & Tea & Cold & 1 \\
\hline & & & & Constipation & 1 \\
\hline & & & & Diuretic & 1 \\
\hline & & & & Hypertension & 1 \\
\hline & & & & Kidney diseases & 5 \\
\hline & & & & Panacea & 1 \\
\hline & & & & $\begin{array}{l}\text { Urinating } \\
\text { problems }\end{array}$ & 1 \\
\hline & & & & Women diseases & 1 \\
\hline Fagaceae & & & & & \\
\hline Populus tremula L., (LJUD054) & асіна, топаль/asina, topal & Bark & Compress & Joint pain & 1 \\
\hline & & & Decoction & Panacea & 1 \\
\hline & & & Tincture & Panacea & 1 \\
\hline & & & Pulverized, eaten & Kidney diseases & 2 \\
\hline & & & Tea & Immune boosting & 1 \\
\hline & & Buds & Tincture & Joint pain & 1 \\
\hline & & Leaves & Topical application & Wounds & 1 \\
\hline & & Sap & Fermented & $\begin{array}{l}\text { Preservative for } \\
\text { birch sap }\end{array}$ & 1 \\
\hline & & Sticks & Put into bed & Aching legs & 1 \\
\hline & & Twigs & Fresh & $\begin{array}{l}\text { Additive to } \\
\text { compote }\end{array}$ & 1 \\
\hline & & & Topical application & Convulses & 1 \\
\hline Quercus robur L., (LUB119) & дуб/dub & Acorns & Dried, grounded & Bread additive & 3 \\
\hline & & & & $\begin{array}{l}\text { Appetizer for } \\
\text { cows }\end{array}$ & 1 \\
\hline & & & Fresh & Bread additive & 1 \\
\hline & & & Milled into flour & $\begin{array}{l}\text { Fodder for home } \\
\text { animals }\end{array}$ & 1 \\
\hline & & Bark & Bath & $\begin{array}{l}\text { Diathesis in } \\
\text { children }\end{array}$ & 1 \\
\hline & & & & Foot hatching & 1 \\
\hline & & & Decoction & Bruises & 1 \\
\hline & & & & Diarrhoea & 3 \\
\hline & & & & Diarrhoea in cows & 2 \\
\hline & & & & Diarrhoea in pigs & 1 \\
\hline & & & & Gingival diseases & 1 \\
\hline & & & & Panacea & 1 \\
\hline & & & & Periodontitis & 1 \\
\hline & & & Dried & Coffee substitute & 1 \\
\hline
\end{tabular}


Table 1 Wild plants used in food, medicinal and veterinary areas (Continued)

\begin{tabular}{|c|c|c|c|c|c|}
\hline & & & & $\begin{array}{l}\text { Taste additive to } \\
\text { strong alcohol }\end{array}$ & 1 \\
\hline & & & & Condiment & 1 \\
\hline & & & & $\begin{array}{l}\text { Condiment for } \\
\text { fermented birch } \\
\text { sap }\end{array}$ & 1 \\
\hline & & & \multirow[t]{2}{*}{ Fresh } & Snack & 1 \\
\hline & & & & $\begin{array}{l}\text { Condiment for } \\
\text { lactofermented } \\
\text { cucumbers }\end{array}$ & 2 \\
\hline & & & Macerated in hot water & Fodder for calves & 1 \\
\hline & & \multirow[t]{6}{*}{ Leaves } & Fresh & $\begin{array}{l}\text { Preservative for } \\
\text { preserve }\end{array}$ & 1 \\
\hline & & & & $\begin{array}{l}\text { Put under bread } \\
\text { when baked }\end{array}$ & 10 \\
\hline & & & & $\begin{array}{l}\text { Condiment for } \\
\text { kvass }\end{array}$ & 1 \\
\hline & & & & $\begin{array}{l}\text { Condiment for } \\
\text { lactofermented } \\
\text { cucumbers }\end{array}$ & 5 \\
\hline & & & & Under bread & 2 \\
\hline & & & Inhalation & Hypertension & 1 \\
\hline & & \multirow{2}{*}{$\begin{array}{l}\text { Malformation } \\
\text { of the leave }\end{array}$} & Juice, topical application & Wounds & 1 \\
\hline & & & Topical application & Warts & 1 \\
\hline & & \multirow[t]{8}{*}{ Twigs } & Bath & Prophylactics & 1 \\
\hline & & & \multirow[t]{3}{*}{ Fresh } & $\begin{array}{l}\text { Preservative for } \\
\text { kvass }\end{array}$ & 3 \\
\hline & & & & $\begin{array}{l}\text { Condiment for } \\
\text { fermented birch } \\
\text { sap }\end{array}$ & 1 \\
\hline & & & & $\begin{array}{l}\text { Condiment for } \\
\text { lactofermented } \\
\text { cucumbers }\end{array}$ & 1 \\
\hline & & & \multirow[t]{4}{*}{ Whisked in sauna } & Back pain & 1 \\
\hline & & & & Healthy & 5 \\
\hline & & & & $\begin{array}{l}\text { Promotes } \\
\text { bloodstream }\end{array}$ & 1 \\
\hline & & & & Prophylactics & 5 \\
\hline \multicolumn{6}{|l|}{ Hydrangeaceae } \\
\hline Philadelphus coronarius L. & жасмін/žasmin & Leaves & Dried & Recreational tea & 1 \\
\hline \multicolumn{6}{|l|}{ Hypericaceae } \\
\hline \multirow{8}{*}{$\begin{array}{l}\text { Hypericum spp., incl. Hypericum } \\
\text { perforatum (LJUB095) }\end{array}$} & \multirow{8}{*}{$\begin{array}{l}\text { светаяннік, зверабой/svietajannik, } \\
\text { zvieraboj }\end{array}$} & \multirow[t]{8}{*}{ Aerial parts } & Bath & Body cleansing & 1 \\
\hline & & & \multirow[t]{4}{*}{ Decoction } & Diarrhoea in cows & 1 \\
\hline & & & & Heart problems & 1 \\
\hline & & & & $\begin{array}{l}\text { Rumination } \\
\text { problems in goats }\end{array}$ & 1 \\
\hline & & & & Scabies & 1 \\
\hline & & & \multirow[t]{3}{*}{ Dried } & $\begin{array}{l}\text { Taste additive to } \\
\text { strong alcohol }\end{array}$ & 2 \\
\hline & & & & Recreational tea & 12 \\
\hline & & & & Condiment & 1 \\
\hline
\end{tabular}


Table 1 Wild plants used in food, medicinal and veterinary areas (Continued)

\begin{tabular}{|c|c|c|c|c|c|}
\hline & & & Fresh & Stomach ache & 1 \\
\hline & & & \multirow[t]{3}{*}{ Tincture, drunk } & $\begin{array}{l}\text { For women to be } \\
\text { strong against } \\
\text { men }\end{array}$ & 1 \\
\hline & & & & Healthy & 3 \\
\hline & & & & Kidney diseases & 1 \\
\hline & & & Smoked & $\begin{array}{l}\text { When piglets do } \\
\text { not go to their } \\
\text { mother }\end{array}$ & 1 \\
\hline & & & \multirow[t]{13}{*}{ Tea } & Cold & 7 \\
\hline & & & & Cough & 3 \\
\hline & & & & Diarrhoea & 2 \\
\hline & & & & Fright & 1 \\
\hline & & & & Heart problems & 4 \\
\hline & & & & Liver diseases & 1 \\
\hline & & & & Panacea & 8 \\
\hline & & & & Pancreas & 1 \\
\hline & & & & Sore throat & 1 \\
\hline & & & & $\begin{array}{l}\text { Stomach } \\
\text { problems }\end{array}$ & 1 \\
\hline & & & & Stomach ache & 4 \\
\hline & & & & Tonus support & 1 \\
\hline & & & & Varix & 2 \\
\hline \multicolumn{6}{|l|}{ Lamiaceae } \\
\hline Comarum palustre L., (LJUB063) & сабельнік/sabieĺnik & Roots & $\begin{array}{l}\text { Tincture, topical } \\
\text { application }\end{array}$ & Joint pain & 3 \\
\hline \multirow[t]{5}{*}{ Origanum vulgare L., (LUUB043) } & \multirow{5}{*}{$\begin{array}{l}\text { мацярдушка, душыца, } \\
\text { мацярдушка/maciarduška, } \\
\text { dušyса }\end{array}$} & \multirow[t]{5}{*}{ Aerial parts } & Dried & Recreational tea & 2 \\
\hline & & & \multirow[t]{4}{*}{ Tea } & Calming & 1 \\
\hline & & & & Cold & 1 \\
\hline & & & & $\begin{array}{l}\text { Stomach } \\
\text { problems }\end{array}$ & 2 \\
\hline & & & & Women diseases & 1 \\
\hline \multirow[t]{4}{*}{ Thymus serpyllum L. (s.I.), (LJUB037) } & \multirow{4}{*}{$\begin{array}{l}\text { верас, чабарок, чабарэц, } \\
\text { чабор/vieras, čabarok, čabarec, } \\
\text { čabor }\end{array}$} & \multirow[t]{4}{*}{ Aerial parts } & Dried & Recreational tea & 8 \\
\hline & & & \multirow[t]{3}{*}{ Tea } & Cold & 3 \\
\hline & & & & Cough & 5 \\
\hline & & & & Hypertension & 1 \\
\hline \multicolumn{6}{|l|}{ Leguminosae } \\
\hline \multirow[t]{3}{*}{ Trifolium spp., (LJUD053) } & \multirow{3}{*}{$\begin{array}{l}\text { канюшына, клевер/kaniušyna, } \\
\text { klievier }\end{array}$} & Aerial parts & Fresh & Fodder for rabbits & 1 \\
\hline & & & & Fodder for pigs & 1 \\
\hline & & Flowers & Tea & Heart diseases & 2 \\
\hline \multicolumn{6}{|l|}{ Melanthiaceae } \\
\hline \multirow[t]{2}{*}{ Veratrum lobelianum Berhn. } & \multirow[t]{2}{*}{ чамярыца/С̌amiaryca } & \multirow[t]{2}{*}{ Aerial parts } & Decoction & $\begin{array}{l}\text { Rumination } \\
\text { problems in cows }\end{array}$ & 1 \\
\hline & & & Fresh & $\begin{array}{l}\text { Rumination } \\
\text { problems in cows }\end{array}$ & 2 \\
\hline \multicolumn{6}{|l|}{ Oleaceae } \\
\hline \multirow[t]{2}{*}{ Syringa vulgaris L., (LJUB071) } & \multirow{2}{*}{$\begin{array}{l}\text { бэз (белы), сірэнь (белая)/bez } \\
\text { (biely), sireń (bielaja) }\end{array}$} & Buds & Tincture & Joint pain & 1 \\
\hline & & Flowers & Decoction & Joint pain & 1 \\
\hline
\end{tabular}


Table 1 Wild plants used in food, medicinal and veterinary areas (Continued)

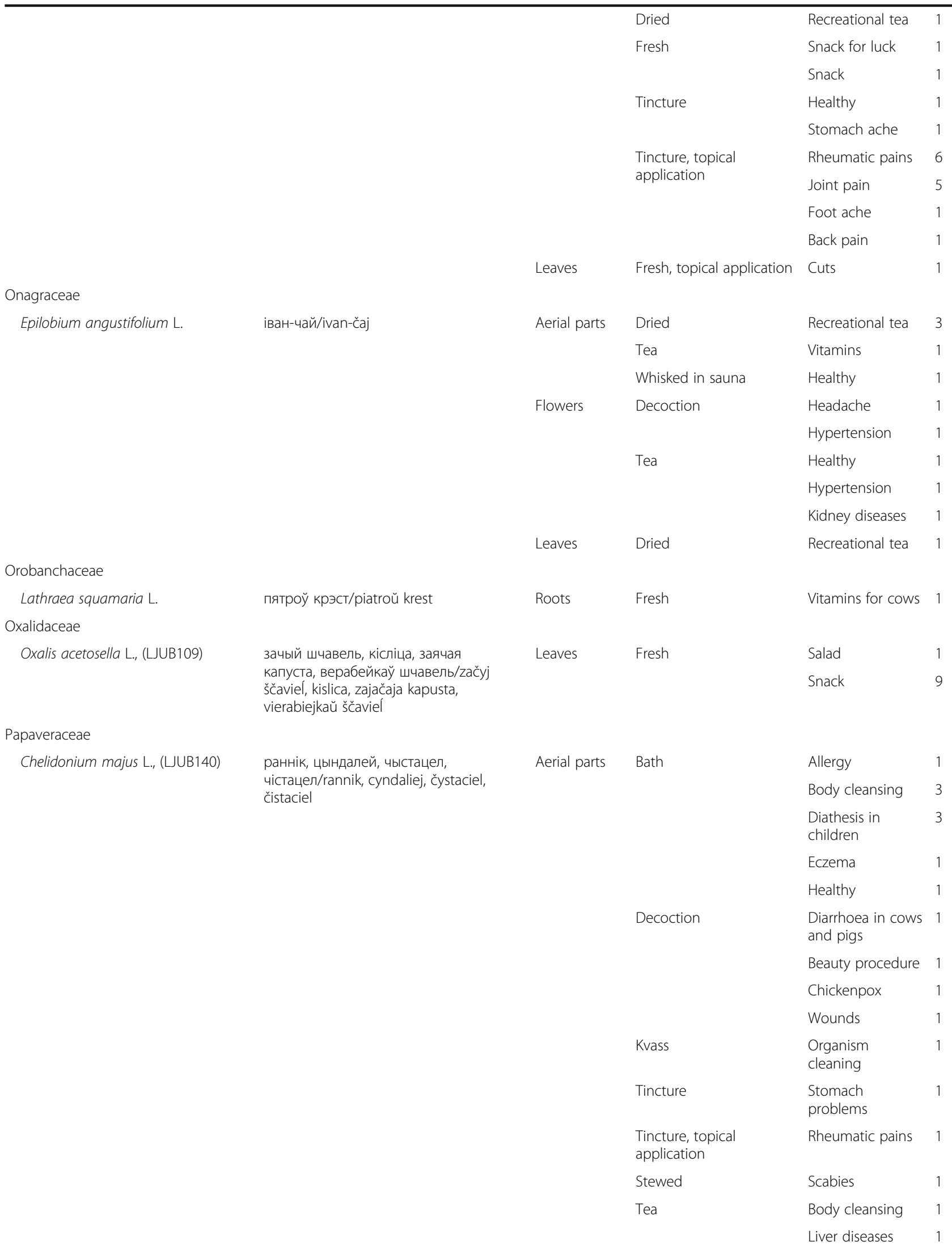


Table 1 Wild plants used in food, medicinal and veterinary areas (Continued)

\begin{tabular}{|c|c|c|c|c|c|}
\hline & & Leaves & Fresh, snacked & Healthy & 1 \\
\hline & & & Topical application & Eczema & 1 \\
\hline & & & & Burns & 2 \\
\hline & & & & Cuts & 3 \\
\hline & & & & Toothache & 1 \\
\hline & & & & Wounds & 4 \\
\hline & & Sap & Diluted juice, lavation & Gingival bleeding & 2 \\
\hline & & & Intake & Gastric ulcer & 1 \\
\hline & & & & Allergy & 1 \\
\hline & & & & Prophylactics & 1 \\
\hline & & & Topical application & Haemostatic & 2 \\
\hline & & & & Skin diseases & 2 \\
\hline & & & & Warts & 9 \\
\hline & & & & Wounds & 9 \\
\hline Papaver spp. & мак, мак-відук, дзікі мак, мак-самасей/ & Seeds & Decoction & Calming & 1 \\
\hline & mak, mak-vıduk, azıkı mak, mak-samasıej & & Dried & Pies & 1 \\
\hline & & & & Soporific & 1 \\
\hline & & & & Condiment & 1 \\
\hline & & & & $\begin{array}{l}\text { Condiment for } \\
\text { bread }\end{array}$ & 1 \\
\hline & & & & $\begin{array}{l}\text { Condiment for } \\
\text { pancakes }\end{array}$ & 1 \\
\hline & & & Fresh & $\begin{array}{l}\text { Helminthic } \\
\text { infection }\end{array}$ & 1 \\
\hline & & & & Pies & 1 \\
\hline & & & & Snack & 1 \\
\hline & & & & Soporific & 1 \\
\hline Pinaceae & & & & & \\
\hline Picea abies (L.) H.Karst., (LJUB121) & елка/jelka & Needles & Foot bath & Joint pain & 1 \\
\hline & & Twigs & Whisked in sauna & Healthy & 1 \\
\hline & & Young cones & Fresh & Jam & 1 \\
\hline & & & Tincture & Healthy & 1 \\
\hline & & & Tea & Cold & 1 \\
\hline Pinus sylvestris L., (LUB082) & сасна, хвоя/sasna, chvoja & Pollen & Whisked in sauna & Healthy & 1 \\
\hline & & Resin & Topical application & Wounds & 1 \\
\hline & & & & Wounds in cows & 1 \\
\hline & & Shoots & Fresh & Jam & 3 \\
\hline & & & Processed & Recreational tea & 1 \\
\hline & & & Tea & Liver diseases & 1 \\
\hline & & & & Lung diseases & 1 \\
\hline & & & & Stomach ache & 1 \\
\hline & & Twigs & Whisked in sauna & Healthy & 1 \\
\hline & & & & Prophylactics & 1 \\
\hline & & Young cones & Decoction & Cold & 1 \\
\hline & & & Fresh & Jam & 3 \\
\hline & & & & Snack & 1 \\
\hline
\end{tabular}


Table 1 Wild plants used in food, medicinal and veterinary areas (Continued)

\begin{tabular}{|c|c|c|c|c|c|}
\hline & & & Jam & Bronchitis & 1 \\
\hline & & & & Cold & 3 \\
\hline & & & & Cough & 1 \\
\hline & & & & Kidney diseases & 1 \\
\hline & & & & Liver diseases & 1 \\
\hline & & & & Sore throat & 1 \\
\hline & & & & Stomach ache & 1 \\
\hline & & & Tincture, topical & Joint pain & 1 \\
\hline & & & applicatıon & Rheumatic pains & 1 \\
\hline & & & Processed & Recreational tea & 2 \\
\hline & & & Tea & Bronchitis & 1 \\
\hline & & & & Cold & 2 \\
\hline & & & & Cough & 1 \\
\hline & & & & Immune boosting & 2 \\
\hline & & & & $\begin{array}{l}\text { Inflammation } \\
\text { processes }\end{array}$ & 2 \\
\hline & & & & Stomach ache & 1 \\
\hline & & & & $\begin{array}{l}\text { Strengthening of } \\
\text { organism }\end{array}$ & 2 \\
\hline & & & & Thyroid glands & 1 \\
\hline & & & & Tuberculosis & 1 \\
\hline Plantaginaceae & & & & & \\
\hline Plantago major L., (LUUB106) & падарожнік/padarožnik & Leaves & Fresh & Salad & 1 \\
\hline & & & & Stomach ache & 1 \\
\hline & & & Juice pressed & Gastric ulcer & 1 \\
\hline & & & Tea & Diarrhoea & 1 \\
\hline & & & & Heart problems & 2 \\
\hline & & & & Low acidity & 1 \\
\hline & & & & Kidney diseases & 1 \\
\hline & & & Topical application & Abscess & 3 \\
\hline & & & & Burns & 1 \\
\hline & & & & Cuts & 4 \\
\hline & & & & $\begin{array}{l}\text { Cuts in } \\
\text { domesticated } \\
\text { animals }\end{array}$ & 1 \\
\hline & & & & Gingival wounds & 1 \\
\hline & & & & Headache & 3 \\
\hline & & & & Haemostatic & 1 \\
\hline & & & & Wounds & 32 \\
\hline Poaceae & & & & & \\
\hline Elymus repens (L.) Gould, (LJUB031) & пырай, пырей, пырэй/pyraj, & Leaves & Fresh & Salad & 1 \\
\hline & & & Topical application & Joint pain & 1 \\
\hline & & Roots & Compress & Eye problems & 1 \\
\hline & & & Decoction & Joint pain & 1 \\
\hline & & & Fresh & Soup & 1 \\
\hline & & & & $\begin{array}{l}\text { Condiment for } \\
\text { salad }\end{array}$ & 1 \\
\hline
\end{tabular}


Table 1 Wild plants used in food, medicinal and veterinary areas (Continued)

\begin{tabular}{|c|c|c|c|c|c|}
\hline & & & $\begin{array}{l}\text { Tincture, topical } \\
\text { application }\end{array}$ & Joint pain & 1 \\
\hline Phleum spp. & цімафееўка, трава/сimafiejeŭka, trava & Aerial parts & Fresh, fodder & $\begin{array}{l}\text { To increase cow } \\
\text { milk production }\end{array}$ & 1 \\
\hline \multicolumn{6}{|l|}{ Polemoniaceae } \\
\hline Polemonium caeruleum L. & сіню́га галубая/siniúha halubaja & Roots & Tincture & Epilepsy & 1 \\
\hline \multicolumn{6}{|l|}{ Polygonaceae } \\
\hline \multirow[t]{3}{*}{ Polygonum aviculare L., (LJUB042) } & \multirow{3}{*}{$\begin{array}{l}\text { спарыш, траўка-мураўка/sparyš, } \\
\text { traŭka-muraŭka }\end{array}$} & \multirow[t]{3}{*}{ Aerial parts } & \multirow[t]{2}{*}{ Tea } & Diarrhoea & 1 \\
\hline & & & & Kidney diseases & 1 \\
\hline & & & Topical application & Headache & 1 \\
\hline \multirow{6}{*}{$\begin{array}{l}\text { Rumex spp. (incl. Rumex acetosa L., } \\
\text { (LJUB151)) }\end{array}$} & \multirow{6}{*}{$\begin{array}{l}\text { шчаўе, шчавель, вераб'іны, шчавель } \\
\text { дзікі, шчавель лясны, шчавер/ščaŭje, } \\
\text { ščavié, ščaviel vierabjiny, ščaviel dziki, } \\
\text { ščavieĺ liasny, ščavier }\end{array}$} & \multirow[t]{6}{*}{ Leaves } & \multirow[t]{4}{*}{ Fresh } & Preserve & 1 \\
\hline & & & & Snack & 14 \\
\hline & & & & Soup & 61 \\
\hline & & & & $\begin{array}{l}\text { Stomach } \\
\text { problems }\end{array}$ & 1 \\
\hline & & & Frozen & Soup & 1 \\
\hline & & & Processed & Soup & 14 \\
\hline Rumex longifolius DC., (LUUB136) & конскі шчавель/konski ščaviel & Seeds & Tea & Diarrhoea & 1 \\
\hline \multicolumn{6}{|l|}{ Rhamnaceae } \\
\hline \multirow[t]{3}{*}{ Frangula alnus Mill., (LJUB015) } & \multirow[t]{3}{*}{ крушына/krušyna } & Bark & Tea & Diarrhoea & 1 \\
\hline & & \multirow[t]{2}{*}{ Fruits } & \multirow[t]{2}{*}{ Fresh } & Constipation & 1 \\
\hline & & & & Dysentery & 2 \\
\hline \multicolumn{6}{|l|}{ Rosaceae } \\
\hline \multirow[t]{8}{*}{ Crataegus spp., (LJUB085) } & \multirow[t]{8}{*}{ баярышнік/bajaryšnik } & \multirow[t]{6}{*}{ Fruits } & Decoction & Heart problems & 1 \\
\hline & & & Dried & $\begin{array}{l}\text { Condiment, } \\
\text { processed birch } \\
\text { sap }\end{array}$ & 1 \\
\hline & & & Tincture & Heart diseases & 1 \\
\hline & & & & Hypertension & 1 \\
\hline & & & Tea & Heart problems & 3 \\
\hline & & & & Hypertension & 1 \\
\hline & & Leaves & Tea & Heart problems & 2 \\
\hline & & $\begin{array}{l}\text { Twigs with } \\
\text { fruits }\end{array}$ & Tincture & Heart problems & 1 \\
\hline \multirow[t]{13}{*}{ Fragaria vesca L., (LJUB048) } & \multirow{13}{*}{$\begin{array}{l}\text { суніцы, земляніка, землянічнік/sunicy, } \\
\text { ziemlianika, ziemlianičnik }\end{array}$} & \multirow[t]{6}{*}{ Aerial parts } & \multirow[t]{2}{*}{ Decoction } & Heart problems & 2 \\
\hline & & & & Kidney diseases & 1 \\
\hline & & & Dried & Recreational tea & 1 \\
\hline & & & Fresh & Recreational tea & 1 \\
\hline & & & \multirow[t]{2}{*}{ Tea } & Liver diseases & 1 \\
\hline & & & & Pneumonia & 1 \\
\hline & & \multirow[t]{7}{*}{ Fruits } & \multirow[t]{3}{*}{ Dried } & Compote & 2 \\
\hline & & & & Liver diseases & 2 \\
\hline & & & & Snack & 1 \\
\hline & & & Fresh & Compote & 5 \\
\hline & & & & Dessert & 2 \\
\hline & & & & Jam & 17 \\
\hline & & & & Snack & 16 \\
\hline
\end{tabular}


Table 1 Wild plants used in food, medicinal and veterinary areas (Continued)

\begin{tabular}{|c|c|c|c|c|c|}
\hline & & & Frozen & Dessert & 1 \\
\hline & & & & Snack & 2 \\
\hline & & & Tea & Cold & 1 \\
\hline & & Leaves & Dried & Recreational tea & 5 \\
\hline & & & Tea & Healthy & 1 \\
\hline Potentilla erecta (L.) Raeusch., & дзівасіл, калган, драўлянка, & Roots & Decoction & Antitoxic & 1 \\
\hline & $\begin{array}{l}\text { lapčatka } \\
\text { lazsil, Kalnan, araullanka, }\end{array}$ & & & Stomach ache & 1 \\
\hline & & & Dried & $\begin{array}{l}\text { Taste additive to } \\
\text { strong alcohol }\end{array}$ & 4 \\
\hline & & & Tincture & Antimicrobic & 1 \\
\hline & & & & Gastric ulcer & 2 \\
\hline & & & & Healthy & 5 \\
\hline & & & & Heart problems & 1 \\
\hline & & & & Inflammation & 1 \\
\hline & & & & Kidney diseases & 3 \\
\hline & & & & Sore throat & 1 \\
\hline & & & & $\begin{array}{l}\text { Stomach } \\
\text { problems }\end{array}$ & 1 \\
\hline & & & & Stomach ache & 12 \\
\hline & & & & Thyroid glands & 1 \\
\hline & & & Tincture, topical & Burns & 1 \\
\hline & & & & Cuts & 1 \\
\hline & & & & Rotten wounds & 1 \\
\hline & & & Tea & Diarrhoea & 1 \\
\hline & & & & Gastritis & 1 \\
\hline & & & & Panacea & 1 \\
\hline & & & & Stomach ache & 4 \\
\hline Prunus padus L., (LJUB084) & чаромха/с̌aromcha & Flowers & Tincture, topical & Joint pain & 1 \\
\hline & & & application & Rheumatic pains & 1 \\
\hline & & Fruits & Compote & Diarrhoea & 1 \\
\hline & & & Fresh & Diarrhoea & 2 \\
\hline & & & & Dysentery & 2 \\
\hline & & & & Snack & 2 \\
\hline & & & Tincture & Diarrhoea & 1 \\
\hline & & Twigs & Placed in water & Disinfectant & 1 \\
\hline Pyrus pyraster (L.) Burgsd., (LJUB050) & грушы дзікія, дзічка/hrušy dzikija, & Fruits & Decoction & Diarrhoea & 5 \\
\hline & dzıcka & & & Diarrhoea in cows & 1 \\
\hline & & & Compote & Cold & 1 \\
\hline & & & & Diarrhoea & 1 \\
\hline & & & & Stomach ache & 1 \\
\hline & & & Dried & Cold & 1 \\
\hline & & & & Compote & 4 \\
\hline & & & & Diarrhoea & 4 \\
\hline & & & & Healthy & 1 \\
\hline & & & & Recreational tea & 1 \\
\hline & & & & Snack & 2 \\
\hline
\end{tabular}


Table 1 Wild plants used in food, medicinal and veterinary areas (Continued)

\begin{tabular}{|c|c|c|c|c|c|}
\hline & & & & Stomach ache & 2 \\
\hline & & & Fermented & Kvass & 2 \\
\hline & & & Fresh & Jam & 1 \\
\hline & & & & Snack & 3 \\
\hline & & & & Fodder for pigs & 1 \\
\hline \multirow[t]{15}{*}{ Rosa spp., (LJUD001) } & \multirow{15}{*}{$\begin{array}{l}\text { шыпшына, шыпоўнік, шыповнік/šypšyna, } \\
\text { šypoŭnik, šypovnik }\end{array}$} & \multirow{15}{*}{ Fruits } & Decoction & Heart problems & 1 \\
\hline & & & \multirow[t]{2}{*}{ Dried } & Recreational tea & 8 \\
\hline & & & & $\begin{array}{l}\text { Condiment for } \\
\text { processed birch } \\
\text { sap }\end{array}$ & 1 \\
\hline & & & \multirow[t]{2}{*}{ Fresh } & Compote & 2 \\
\hline & & & & Jam & 3 \\
\hline & & & Tincture & Stomach ache & 1 \\
\hline & & & \multirow[t]{9}{*}{ Tea } & Cold & 2 \\
\hline & & & & Healthy & 1 \\
\hline & & & & Heart diseases & 1 \\
\hline & & & & Hypertension & 1 \\
\hline & & & & Immune boosting & 3 \\
\hline & & & & $\begin{array}{l}\text { Inflammation } \\
\text { processes }\end{array}$ & 2 \\
\hline & & & & Kidney diseases & 3 \\
\hline & & & & Liver diseases & 2 \\
\hline & & & & Panacea & 2 \\
\hline \multirow[t]{14}{*}{ Rubus caesius L., (LJUB003) } & \multirow{14}{*}{$\begin{array}{l}\text { ажыны, ежавіка (лесная)/ažyny, } \\
\text { ježavika (liesnaja) }\end{array}$} & \multirow[t]{11}{*}{ Fruits } & \multirow[t]{7}{*}{ Fresh } & Compote & 2 \\
\hline & & & & Dessert & 2 \\
\hline & & & & Jam & 14 \\
\hline & & & & Kissel & 1 \\
\hline & & & & $\begin{array}{l}\text { Taste additive to } \\
\text { strong alcohol }\end{array}$ & 1 \\
\hline & & & & Recreational tea & 1 \\
\hline & & & & Snack & 8 \\
\hline & & & \multirow[t]{3}{*}{ Frozen } & Hypertension & 1 \\
\hline & & & & Liver diseases & 1 \\
\hline & & & & Snack & 2 \\
\hline & & & Processed & Pies & 1 \\
\hline & & Leaves & Dried & Recreational tea & 3 \\
\hline & & Twigs & Dried & Recreational tea & 1 \\
\hline & & $\begin{array}{l}\text { Twigs with } \\
\text { fruits }\end{array}$ & Dried & Recreational tea & 1 \\
\hline \multirow[t]{8}{*}{ Rubus idaeus L., (LUB044) } & \multirow{8}{*}{$\begin{array}{l}\text { маліна (лясная), маліннік/malina } \\
\text { (liasnaja), malinnik }\end{array}$} & \multirow[t]{6}{*}{ Aerial parts } & Dried & Recreational tea & 7 \\
\hline & & & Fresh & Snack & 1 \\
\hline & & & \multirow[t]{4}{*}{ Tea } & Cold & 1 \\
\hline & & & & Cough & 1 \\
\hline & & & & Healthy & 1 \\
\hline & & & & Sore throat & 2 \\
\hline & & \multirow[t]{2}{*}{ Fruits } & Compote & Cough & 1 \\
\hline & & & Dried & Cold & \\
\hline
\end{tabular}


Table 1 Wild plants used in food, medicinal and veterinary areas (Continued)

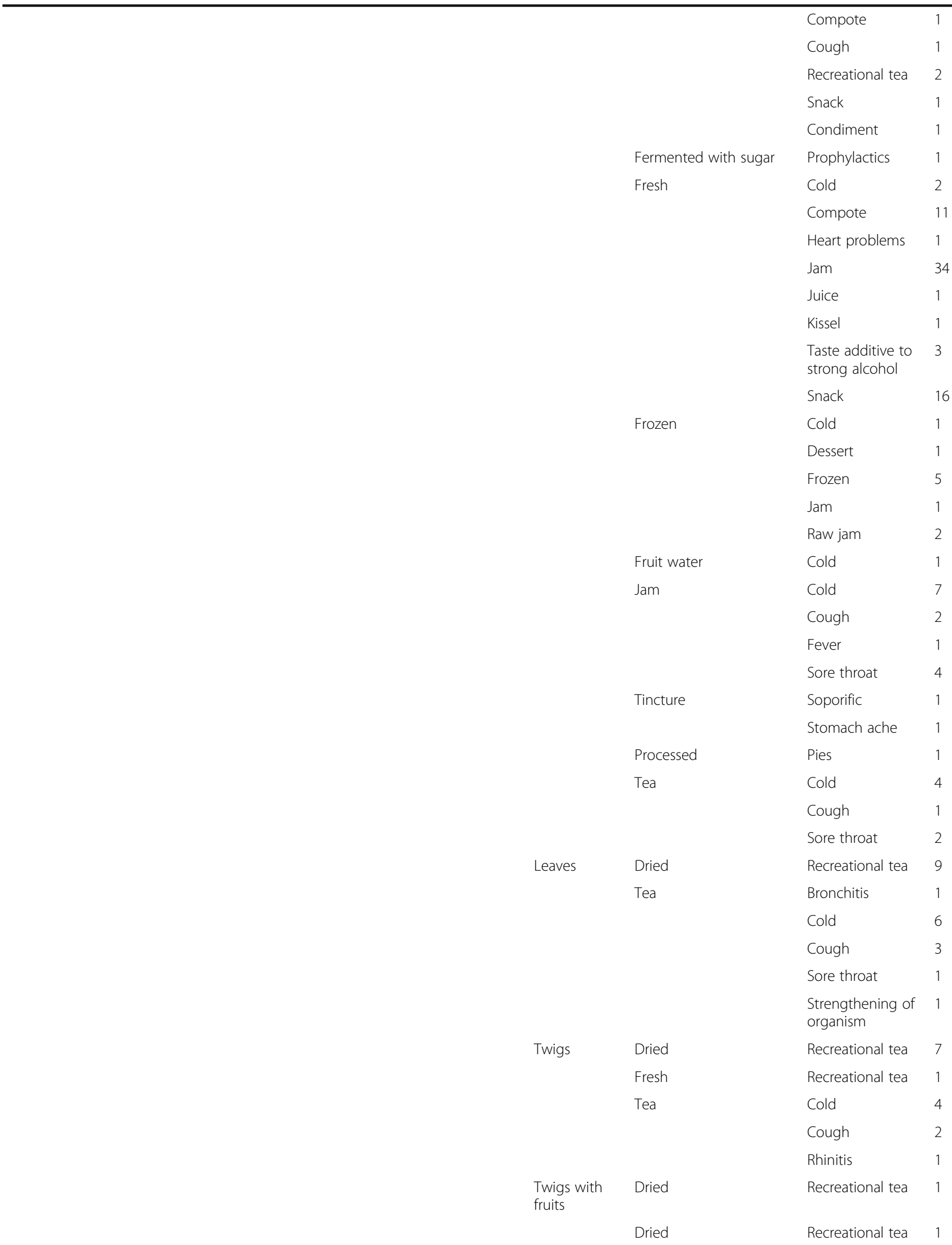


Table 1 Wild plants used in food, medicinal and veterinary areas (Continued)

\begin{tabular}{|c|c|c|c|c|c|}
\hline & & Twigs with & Tea & Cold & 3 \\
\hline & & & & Constipation & 1 \\
\hline & & & & Cough & 1 \\
\hline & & & & Pneumonia & 1 \\
\hline Rubus saxatilis $\mathrm{L}$. & $\begin{array}{l}\text { камяніка, касцяніка/kamianika, } \\
\text { kascianika }\end{array}$ & Fruits & Fresh & Snack & 2 \\
\hline Sorbus aucuparia L., (LUB075) & арабіна, красная рабіна/arabina, & Flowers & Tea & Cold & 1 \\
\hline & & Fruits & Decoction & $\begin{array}{l}\text { Bone } \\
\text { strengthening }\end{array}$ & 1 \\
\hline & & & Dried & Hypotension & 1 \\
\hline & & & & Recreational tea & 1 \\
\hline & & & Fermented & Wine & 1 \\
\hline & & & Fermented with sugar & Prophylactics & 1 \\
\hline & & & Fresh & Cold & 1 \\
\hline & & & & Jam & 2 \\
\hline & & & & Snack & 1 \\
\hline & & & & Syrup & 1 \\
\hline & & & Frozen on twigs & CO-intoxication & 1 \\
\hline & & & Raw jam & Cough & 2 \\
\hline & & & Tincture & Healthy & 2 \\
\hline & & Leaves & Tea & Hypotension & 1 \\
\hline Salicaceae & & & & & \\
\hline Salix spp. & вярба свечаная, іва/viarba & Seeds & Massage & Prophylactics & 1 \\
\hline & sviecanaja, ıva & Shoots & Tea & Cold & 1 \\
\hline Santalaceae & & & & & \\
\hline Viscum album L., (LJUB074) & амяла/amiala & Leaves & Fresh & $\begin{array}{l}\text { Organism } \\
\text { cleansing }\end{array}$ & 1 \\
\hline Sapindaceae & & & & & \\
\hline Acer platanoides L., (LJUB131) & клён/klion & Leaves & Fresh & Put under bread & 4 \\
\hline & & & $\begin{array}{l}\text { Dried, from twigs } \\
\text { blessed on Pentecost, } \\
\text { topical application }\end{array}$ & Wounds & 2 \\
\hline & & Sap & Boiled into syrup & $\begin{array}{l}\text { Fodder for bees } \\
\text { in spring }\end{array}$ & 1 \\
\hline & & & Fermented & Kvass & 2 \\
\hline & & & Fresh & Drink & 10 \\
\hline & & & & Snack & 3 \\
\hline & & & Processed & Drink & 1 \\
\hline & & Twigs & Presence in house & Aroma therapy & 1 \\
\hline & & & Whisked in sauna & Healthy & 1 \\
\hline & & & & Prophylactics & 2 \\
\hline Aesculus hippocastanum L., & каштан/kaštan & Flowers & Decoction & Joint pain & 1 \\
\hline & & & Tincture, topical & Foot ache & 2 \\
\hline & & & & Joint pain & 3 \\
\hline & & & & Rheumatic pains & 2 \\
\hline & & Fruits & Tincture, topical & Rheumatic pains & 1 \\
\hline & & & & Joint pain & 2 \\
\hline
\end{tabular}


Table 1 Wild plants used in food, medicinal and veterinary areas (Continued)

\begin{tabular}{|c|c|c|c|c|c|}
\hline & & Pericarp & Decoction & Varix & 2 \\
\hline \multicolumn{6}{|l|}{ Scrophulariaceae } \\
\hline \multirow[t]{8}{*}{ Verbascum thapsus L., (LUUB062) } & \multirow[t]{8}{*}{ дзівасіл/dzivasil } & \multirow[t]{4}{*}{ Aerial parts } & Decoction & Panacea & 1 \\
\hline & & & Tincture & Liver diseases & 1 \\
\hline & & & $\begin{array}{l}\text { Tincture, topical } \\
\text { application }\end{array}$ & Joint pain & 1 \\
\hline & & & Tea & Lung diseases & 1 \\
\hline & & Flowers & Tea & $\begin{array}{l}\text { Stomach } \\
\text { problems }\end{array}$ & 1 \\
\hline & & \multirow[t]{2}{*}{ Leaves } & Tincture & Stomach ache & 1 \\
\hline & & & Tea & Stomach ache & 1 \\
\hline & & Roots & Tea & Healthy & 1 \\
\hline \multicolumn{6}{|l|}{ Solanaceae } \\
\hline Datura stramonium L. & шалянец, дурман/šalianiec, durman & Fruits & Tincture & Diarrhoea & 1 \\
\hline \multicolumn{6}{|l|}{ Tiliaceae } \\
\hline \multirow[t]{17}{*}{ Tilia cordata Mill., (LUB089) } & \multirow[t]{17}{*}{ ліпа/lipa } & Buds & Decoction & Cold & 2 \\
\hline & & Cambium & Boiled in milk & Abscess & 1 \\
\hline & & \multirow[t]{11}{*}{ Flowers } & \multirow[t]{2}{*}{ Dried } & $\begin{array}{l}\text { Taste additive to } \\
\text { strong alcohol }\end{array}$ & 1 \\
\hline & & & & Recreational tea & 32 \\
\hline & & & \multirow[t]{2}{*}{ Fresh } & $\begin{array}{l}\text { Taste additive to } \\
\text { strong alcohol }\end{array}$ & 1 \\
\hline & & & & Rhinitis & 1 \\
\hline & & & \multirow[t]{7}{*}{ Tea } & Cold & 17 \\
\hline & & & & Cough & 7 \\
\hline & & & & Diarrhoea & 1 \\
\hline & & & & Healthy & 3 \\
\hline & & & & Headache & 1 \\
\hline & & & & Hypertension & 1 \\
\hline & & & & Sore throat & 1 \\
\hline & & \multirow[t]{2}{*}{ Leaves } & Dried & Recreational tea & 1 \\
\hline & & & Tea & Healthy & 1 \\
\hline & & \multirow[t]{2}{*}{ Twigs } & \multirow[t]{2}{*}{ Whisked in sauna } & Cold & 1 \\
\hline & & & & Healthy & 1 \\
\hline \multicolumn{6}{|l|}{ Typhaceae } \\
\hline Typha spp. & рагоз, камыш/rahoz, kamyš & Fuzz & Topical application & Burns & 3 \\
\hline \multicolumn{6}{|l|}{ Urticaceae } \\
\hline \multirow[t]{6}{*}{ Urtica dioica L., (LUUB087) } & \multirow[t]{6}{*}{ крапіва/krapiva } & \multirow[t]{6}{*}{ Aerial parts } & \multirow[t]{5}{*}{ Decoction } & Fodder for cows & 1 \\
\hline & & & & Hair care & 6 \\
\hline & & & & $\begin{array}{l}\text { Strengthening of } \\
\text { piglets }\end{array}$ & 1 \\
\hline & & & & $\begin{array}{l}\text { Thickening of } \\
\text { blood }\end{array}$ & 1 \\
\hline & & & & Washing hair & 1 \\
\hline & & & Dried & Fodder for cows & 1 \\
\hline
\end{tabular}


Table 1 Wild plants used in food, medicinal and veterinary areas (Continued)

\begin{tabular}{|c|c|c|c|c|c|}
\hline & & & Fresh & Salad & 3 \\
\hline & & & & Snack & 1 \\
\hline & & & & Soup & 17 \\
\hline & & & & Fodder for cows & 1 \\
\hline & & & & $\begin{array}{l}\text { Strengthening of } \\
\text { pigs }\end{array}$ & 3 \\
\hline & & & Processed & Soup & 1 \\
\hline & & & Topical application & Back pain & 1 \\
\hline & & Leaves & Decoction & Hair care & 3 \\
\hline & & & Dried & $\begin{array}{l}\text { Condiment for } \\
\text { soup }\end{array}$ & 1 \\
\hline & & & Fresh & Bread additive & 1 \\
\hline & & & & Cutlets & 1 \\
\hline & & & & Heart problems & 1 \\
\hline & & & & $\begin{array}{l}\text { Organism } \\
\text { cleansing }\end{array}$ & 1 \\
\hline & & & & Salad & 4 \\
\hline & & & & Snack & 4 \\
\hline & & & & Soup & 19 \\
\hline & & & & $\begin{array}{l}\text { Condiment for } \\
\text { salad }\end{array}$ & 1 \\
\hline & & & $\begin{array}{l}\text { Scaled, topical } \\
\text { application }\end{array}$ & Rheumatic pains & 1 \\
\hline & & & Tea & Diabetes & 2 \\
\hline & & & & Urinary bladder & 1 \\
\hline & & & & Vesical cleansing & 1 \\
\hline & & & & Women diseases & 1 \\
\hline & & & Washing & Hair care & 1 \\
\hline & & Seeds & Fresh & Soup & 2 \\
\hline Urtica urens L. & крапіва пякучка, крапіва & Aerial parts & Decoction & Hair care & 1 \\
\hline & $\begin{array}{l}\text { Жыжка/krapıva plakucka, } \\
\text { krapiva žyžka }\end{array}$ & & Fresh & Fodder for chicks & 1 \\
\hline & & & & $\begin{array}{l}\text { Preservation of } \\
\text { food }\end{array}$ & 2 \\
\hline & & & & Soup & 4 \\
\hline & & & & Fodder for turkey & 1 \\
\hline Violaceae & & & & & \\
\hline Viola arvensis Murray, (LUB009) & браткі, іван-ды-мар'я, браткі/bratki, & Aerial parts & Tea & Fever & 1 \\
\hline & Ivan-dy-marja & Flowers & Bath & $\begin{array}{l}\text { Diathesis in } \\
\text { children }\end{array}$ & 1 \\
\hline & & & Dried & Recreational tea & 1 \\
\hline & & & Topical application & Earache & 1 \\
\hline & & Leaves & Tea & Cold & 1 \\
\hline & & & Topical application & Wounds & 1 \\
\hline & & Roots & Tea & Panacea & 1 \\
\hline
\end{tabular}




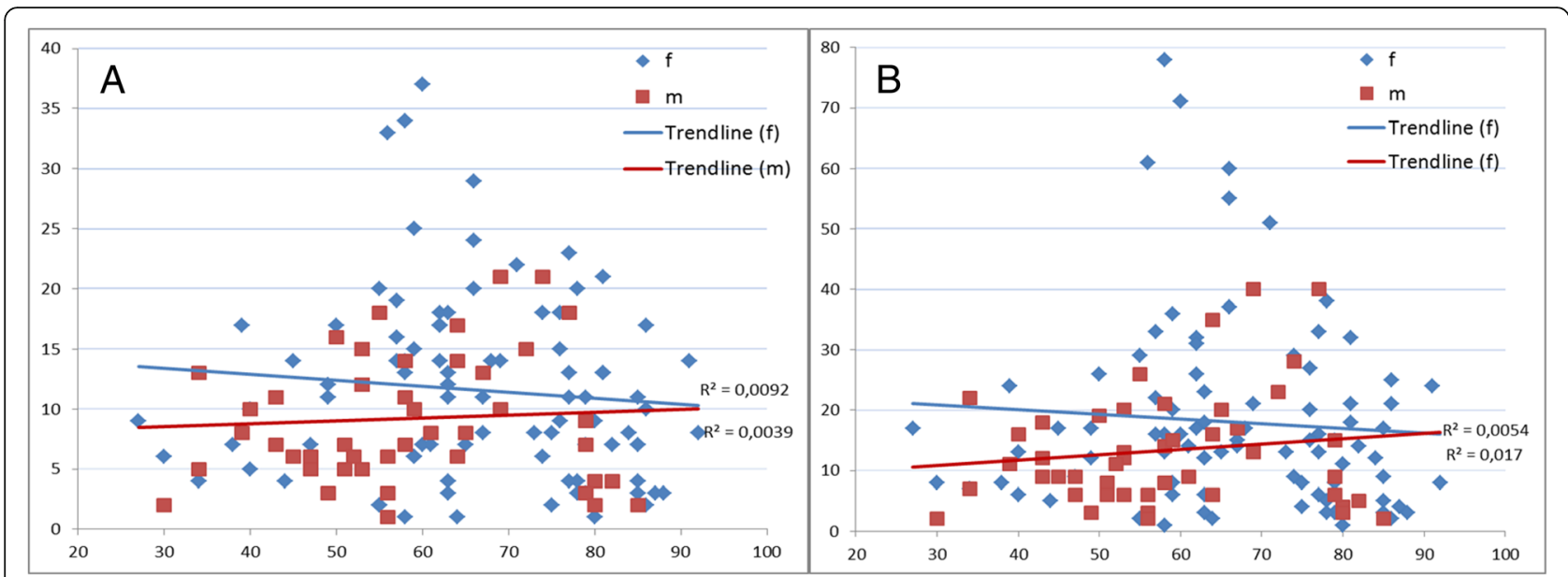

Fig. 2 Distribution of the number of taxa used (a) and DUR (b) according to the age and sex of interviewees

and Urtica dioica), followed by compote ( $\mathrm{FIC}=0.88$ ), dominated by two forest berries (Vaccinium uliginosum and Vaccinium myrtillus), and condiments $(\mathrm{FIC}=0.84$ ) dominated by Armoracia rusticana, Quercus robur, Carum carvi and Vaccinium oxycoccos.

The most homogenous general category was jam, which possessed one of the highest FIC values (0.94). Eight of its 14 taxa, all but one of them (Taraxacum officinale) forest berries, were used by over 10 interviewees. Quite equivocal is the category of recreational tea, which contained the highest number of taxa (29) and a relatively high FIC (0.81): five taxa (Tilia cordata, Rubus idaeus, Vaccinium vitis-idaea, Hypericum spp., Vaccinium myrtillus) were mentioned by 10 or more interviewees, while 18 taxa were mentioned by only one or two respondents. A similar pattern was observed for snacks $(\mathrm{FIC}=0.87$ ), which is highly dominated by fruits (the most numerous of them being Vaccinium myrtillus, Vaccinium oxycoccos, Vaccinium uliginosum, Fragaria vesca, and Rubus idaeus), but also contains a wide variety of occasionally mentioned taxa.

\section{Comparison with data known about the 20th-21st centuries in food area}

The number of taxa (67) outlined in [10] is greater than that recorded in this study (58). However, as we excluded from the list fruiting trees and shrubs that are mainly collected from cultivation, the difference in numbers is below $10 \%$. The difference in taxa used is remarkable for snacks, which are well represented in [10], but rather rare in the present study. Several other uses such as Matricaria spp. and Artemisia absinthium listed in [10] as recreational teas, were used in this region, solely as medicine. In addition, Berberis vulgaris, for example, was not used, but we also did not encounter any shrubs in the region. For some taxa, the difference was on the level of different species from the same genus (Viola).

More than ten taxa were added to the known modern uses of recreational teas in Belarus by the results of this study. Of these, the reliability criterion was met by seven taxa (in order of popularity): Vaccinium vitis-idaea, $\mathrm{Hy}$ pericum spp., Vaccinium myrtillus, Fragaria vesca, Rubus caesius, Epilobium angustifolium, and Pinus sylvestris. We also documented the use of Prunus padus and Maianthemum bifolium as snacks, which have been observed elsewhere (for example in Estonia [58, 59]), but not in Belarus.

We recorded some uses which were absent in recent data, but present in historical sources, such as various uses of the sap of Acer platanoides, or the use of Papaver spp. in bread and sweets. Also, the use of Chenopodium album in soups is solely referred to as "former" in [10], but this study showed that it is still used, although less intensely than in the past.

\section{Medicinal area}

Medicinal use was reported for 74 taxa belonging to 38 families. Of these, only 16 taxa were mentioned by more than $10 \%$ of the interviewees, while 19 taxa were mentioned by less than three people; the reliability criterion was met by $74.3 \%$ (55) of the taxa. The most diversely used families were Rosaceae (9 taxa/178 DUR), Asteraceae (12 taxa/151 DUR), Ericaceae (7 taxa/108 DUR), Betulaceae (2 taxa/72 DUR), Papaveraceae (2 taxa/61 DUR) and Plantaginaceae (1 taxa/52 DUR). The ten most popular taxa in this area differed from the overall top list considerably more than did wild food plants: Betula spp., Rubus idaeus, Vaccinium myrtillus, Chelidonium majus, Plantago major, Hypericum spp., Potentilla erecta, Tilia cordata, Arctium tomentosum, and Quercus robur. 
Table 2 General categories in food area, their compostition and Use Citations

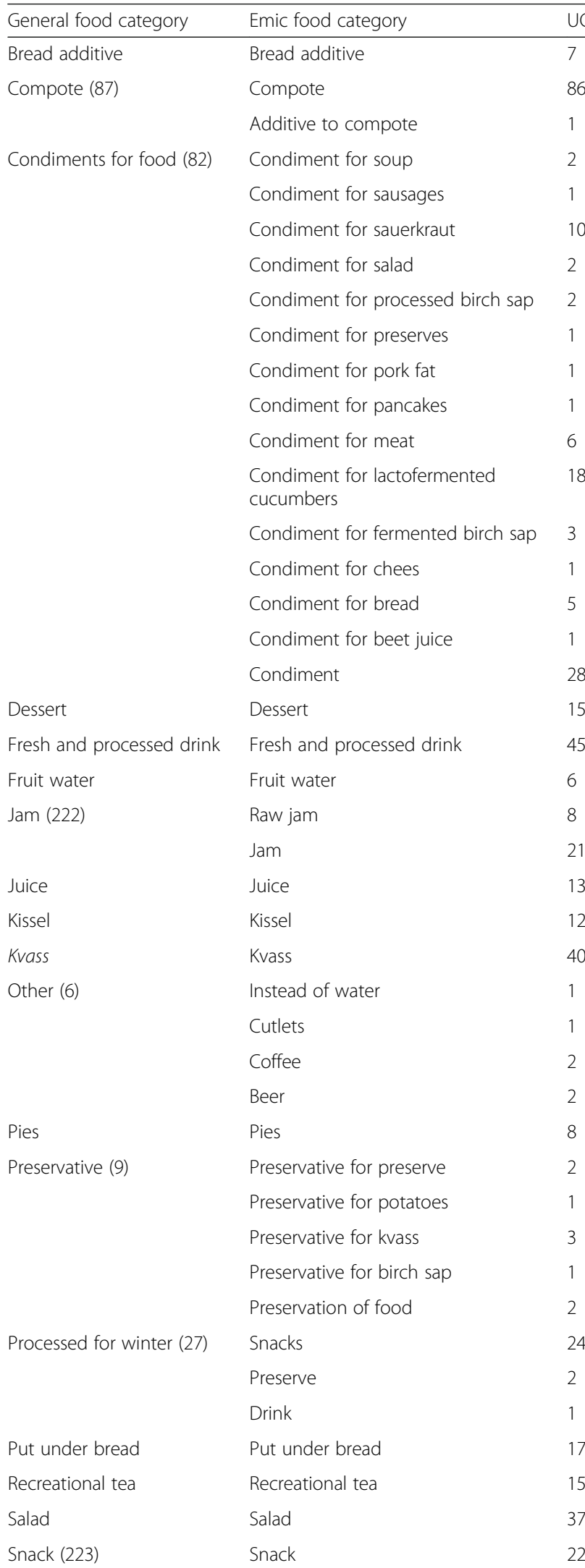

Table 2 General categories in food area, their compostition and Use Citations (Continued)

\begin{tabular}{llc}
\hline & Ritual snack & 1 \\
Soup & Soup & 160 \\
Syrup & Syrup & 5 \\
Taste additive to strong & Taste additive to strong alcohol & 35 \\
alcohol & & \\
Wine & Wine & 4 \\
\hline
\end{tabular}

The medicinal area contained 15 taxa not used in the food area, which were named by more than three interviewees. Only one of these (Tanacetum vulgare), however, was mentioned by more than $10 \%$ of interviewees (14). The majority of the other 14 taxa (Tussilago farfara, Aesculus hippocastanum, Achillea millefolium, Matricaria spp., Juniperus communis, Bidens tripartita, Verbascum thapsus, Alnus spp., Frangula alnus, Trifolium spp., Equisetum arvense, Comarum palustre, Typha spp., Polygonum aviculare) are common and well-known medicinal plants in the former Soviet states.

Among the 113 respondents who claimed to have used wild plants in the medicinal area, the three most knowledgeable used 29, 27 and 26 taxa, numbering 42, 34 and 54 DUR, respectively. Eleven people named only one use of wild plants in the medicinal area. The mean number of used taxa was 6.2 and the mean number of DUR was 8.6.

All the DUR in the medicinal area were distributed between 122 emic categories, which can be attributed to 16 general categories related to health and wellbeing (Table 3). However, $85 \%$ of all DUR are distributed between six general categories (Fig. 4).

The Informant Consensus Factor for the whole medicinal area was 0.92 (698 use citations for 74 taxa).

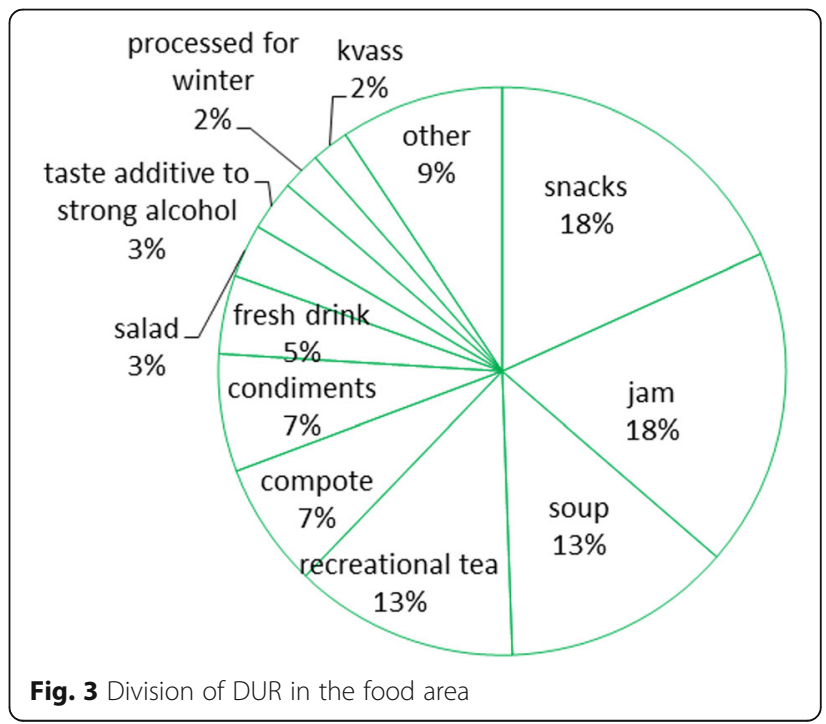


Table 3 General categories in medicinal area, included emic categories and Use Citations

\begin{tabular}{|c|c|}
\hline $\begin{array}{l}\text { General categories in medicinal area } \\
\text { (sum of DUR) }\end{array}$ & Emic disease categories \\
\hline \multirow[t]{11}{*}{ Cardiovascular (82) } & Vesical cleaning \\
\hline & Varix \\
\hline & Thickening of blood \\
\hline & Promotes bloodstream \\
\hline & Hypotension \\
\hline & Hypertension \\
\hline & Haemostatic \\
\hline & Heart problem \\
\hline & Heart disease \\
\hline & Bad blood \\
\hline & Anaemia \\
\hline \multirow[t]{4}{*}{ Cosmetics (23) } & Rejuvenating \\
\hline & Hair care \\
\hline & Dandruff \\
\hline & Beauty procedure \\
\hline \multirow[t]{3}{*}{ Culture bound (3) } & Fright \\
\hline & $\begin{array}{l}\text { For women to be strong } \\
\text { against men }\end{array}$ \\
\hline & Evil eye \\
\hline \multirow[t]{13}{*}{ Dermatological (98) } & Wounds \\
\hline & Warts \\
\hline & Tumour \\
\hline & Skin diseases \\
\hline & Scabies \\
\hline & Rotten wounds \\
\hline & Inflammation processes \\
\hline & Foot hatching \\
\hline & Eczema \\
\hline & Cuts \\
\hline & Burns \\
\hline & Allergy \\
\hline & Abscess \\
\hline \multirow[t]{3}{*}{ Endocrinological (8) } & Thyroid glands \\
\hline & Pancreas \\
\hline & Diabetes \\
\hline \multirow[t]{8}{*}{ Gastrointestinal (180) } & Stomach ache \\
\hline & Stomach problems \\
\hline & Low acidity \\
\hline & Liver diseases \\
\hline & Haemorrhoids \\
\hline & Gastritis \\
\hline & Gastric ulcer \\
\hline & Gall stones \\
\hline
\end{tabular}

Table 3 General categories in medicinal area, included emic categories and Use Citations (Continued)

\begin{tabular}{|c|c|c|}
\hline & Dysentery & 4 \\
\hline & Diarrhoea & 63 \\
\hline & Constipation & 4 \\
\hline & Bile neutralizer & 1 \\
\hline & Bile deficiency & 1 \\
\hline \multirow[t]{25}{*}{ General health (182) } & Vitamins & 4 \\
\hline & Washing hair & 1 \\
\hline & Tonus support & 1 \\
\hline & Tonic & 1 \\
\hline & Tick prevention & 1 \\
\hline & Strengthening of organism & 4 \\
\hline & Prophylactics & 26 \\
\hline & Panacea & 32 \\
\hline & Pain & 1 \\
\hline & Organism cleansing & 7 \\
\hline & Inflammation processes & 5 \\
\hline & Immune boosting & 7 \\
\hline & Healthy & 55 \\
\hline & Hangover & 1 \\
\hline & Foot ache & 1 \\
\hline & Fever & 3 \\
\hline & Disinfectant & 3 \\
\hline & Diathesis in children & 12 \\
\hline & CO-intoxication & 2 \\
\hline & Cancer & 3 \\
\hline & Bone strengthening & 1 \\
\hline & Body cleansing & 5 \\
\hline & Aroma therapy & 1 \\
\hline & Appetizer & 3 \\
\hline & Allergy & 2 \\
\hline \multirow[t]{2}{*}{ Gynaecological (7) } & Women diseases & 6 \\
\hline & $\begin{array}{l}\text { To increase milk production } \\
\text { in woman }\end{array}$ & 1 \\
\hline \multirow[t]{5}{*}{ Infection (14) } & Tuberculosis & 2 \\
\hline & Scabies & 1 \\
\hline & Helminthic infection & 9 \\
\hline & Chickenpox & 1 \\
\hline & Anti-microbic & 1 \\
\hline \multirow[t]{6}{*}{ Musculoskeletal (91) } & Joint pain & 50 \\
\hline & Rheumatic pains & 17 \\
\hline & Knee ache & 2 \\
\hline & Adhesion of bones & 1 \\
\hline & Foot ache & 7 \\
\hline & Bruises & 3 \\
\hline
\end{tabular}


Table 3 General categories in medicinal area, included emic categories and Use Citations (Continued)

\begin{tabular}{|c|c|c|}
\hline & Painful place & 3 \\
\hline & Back pain & 7 \\
\hline & Aching legs & 1 \\
\hline \multirow[t]{5}{*}{ Nephrological and urological (40) } & Urinating problems & 1 \\
\hline & Urinary bladder & 2 \\
\hline & Kidney stones & 1 \\
\hline & Kidney diseases & 29 \\
\hline & Diuretic & 7 \\
\hline \multirow[t]{8}{*}{ Neurological (29) } & Soporific & 3 \\
\hline & Sedative & 1 \\
\hline & Nerves & 2 \\
\hline & Insomnia & 1 \\
\hline & Headache & 11 \\
\hline & Epilepsy & 4 \\
\hline & Convulses & 1 \\
\hline & Calming & 6 \\
\hline \multirow[t]{2}{*}{ Ophthalmological (21) } & Improve vision & 5 \\
\hline & Eye problems & 16 \\
\hline \multirow[t]{6}{*}{ Oral and dental (7) } & Toothache & 1 \\
\hline & Teething pain in children & 1 \\
\hline & Periodontitis & 1 \\
\hline & Gingival diseases & 1 \\
\hline & Gingival bleeding & 2 \\
\hline & Gingival wound & 1 \\
\hline Outer influence (1) & Antitoxic & 1 \\
\hline \multirow[t]{9}{*}{ Respiratory (172) } & Sore throat & 22 \\
\hline & Rhinitis & 2 \\
\hline & Pneumonia & 2 \\
\hline & Lung diseases & 2 \\
\hline & Expectorant & 1 \\
\hline & Earache & 1 \\
\hline & Cough & 42 \\
\hline & Cold & 94 \\
\hline & Bronchitis & 6 \\
\hline
\end{tabular}

All general categories in the medicinal area had lower FIC values and only seven general categories had a FIC over 0.7.

The category of respiratory illnesses with a FIC value of 0.84 (26 taxa) was clearly dominated by two taxa: $R u$ bus idaeus and Tilia cordata, both used primarily to treat cold, cough and sore throat; two more taxa ( $H y$ pericum spp. and Pinus sylvestris) were named by more than 10 people and used mainly against cold. Among the rest there were three taxa used in a certain mode that met the reliability criterion: tea made from the aerial

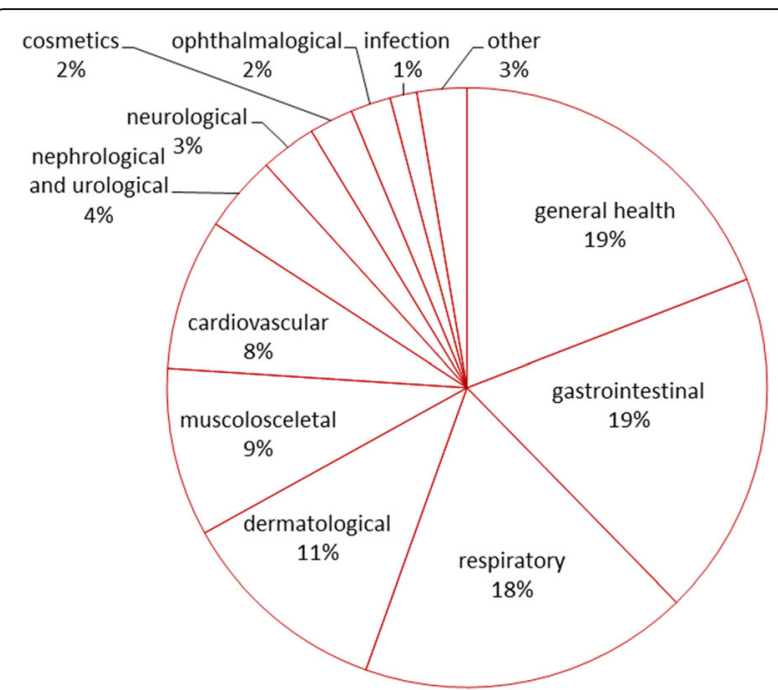

Fig. 4 Division of DUR in the medicinal area

parts of Thymus serpyllum and Ledum palustre or leaves of Tussilago farfara to alleviate cough; $54 \%$ of taxa in the respiratory category were used by less than three respondents.

The category of ophthalmological diseases had a similarly high FIC (0.84), but one of the smallest use citation numbers (20). This category also had a very small number of used taxa (four), dominated by one taxon: Vaccinium myrtillus, which is considered good for eye problems and believed to improve vision.

Dermatological diseases $(\mathrm{FIC}=0.81)$ contained 21 taxa, two of which were dominant: Plantago major with the well-known topical application of fresh leaves on wounds, burns, cuts and abscesses, and Chelidonium majus with a wide variety of applications and modes of use, including the topical application of sap on wounds (said to be welded) and warts, as well as treating other skin diseases and injuries (like eczema, cuts, burns) by applying leaves, and a decoction of the aerial parts for external application to treat eczema and also washing wounds. As for the other taxa uses in the dermatology category, the reliability criterion was met only by the use of a tincture made from the buds of Betula spp. for cleansing wounds and the topical application of the fuzz of Typha spp. to treat burns. All of the remaining taxa used in the dermatological category (including the internationally wellknown haemostatic Achillea millefolium) were mentioned by less than five people and did not meet the reliability criterion for a specific application mode.

The gastrointestinal category $(\mathrm{FIC}=0.77)$ contained 37 taxa (almost half of them were mentioned by less than three respondents). The fruits of two of the three most dominant taxa, Vaccinium myrtillus and Pyrus pyraster, were utilized in a wide variety of use modes 
mainly to treat diarrhoea. A tincture made from the roots of Potentilla erecta was primarily used to alleviate stomach ache. Of the remaining taxa-use combinations, only a few met the reliability criterion: seven taxa used against three emic disease categories. The most common of these was diarrhoea, which was treated with a tea or decoction of the aerial parts of Artemisia absinthium and Tanacetum vulgare, the cone-like fruits of Alnus spp., and the bark of Quercus robur as well as a tincture made from the buds or catkins of Betula spp. The other emic disease categories that met the reliability criterion were stomach ache, which was treated with tea made from the aerial parts of Hypericum spp. and a tincture of Betula spp., and the use of a tea made from Helichrysum arenarium to treat liver disease.

The category of general health $(\mathrm{FIC}=0.75)$ contained the highest number of taxa in one general category (46). It was dominated by four taxa: Betula spp. (used mainly as a prophylactic and healthy whisking in sauna, but also a tincture made from the buds as a panacea), Hypericum spp. (tea as a panacea), Quercus robur L. (wellbeing and prophylactic whisking in sauna) and Chelidonium majus (decoction used as a bath against diathesis in children and for body cleansing). Of the other combinations of taxa and uses, the reliability criterion was met by four taxa used on three occasions: an alcoholic infusion of the roots of Potentilla erecta and a tea made from the inflorescences of Tilia cordata were considered healthy, a bath of the decoction of Bidens tripartita was used against diathesis in children and a tea made from the fruits of Rosa spp. for boosting the immune system.

The musculoskeletal category $(\mathrm{FIC}=0.74$ ) contained 22 taxa, three of which were used by more than ten people. The fresh leaves of Arctium tomentosum were mainly topically applied to alleviate joint and foot pain. A tincture of Syringa vulgaris was applied on rheumatic and joint pains and a tincture made from the inflorescences or fruits of Aesculus hippocastanum was used for the same purpose. Of the remaining taxa only two more met the reliability criterion: Taraxacum officinale was used in a variety of modes, and a tincture of Comarum palustre topically applied to alleviate joint pain.

Within other general categories, only a few uses met the reliability criterion. In the cardiovascular category this included a tincture made from the fruits of Viburnum opulus used against hypertension, as well as a tea or decoction of the aerial parts or roots of Valeriana officinalis and a tea made from the fruits of Crataegus spp. or the areal parts of Hypericum spp. to treat heart problems. In the nephrological and urological category the leaves of Vaccinium vitis-idaea or the fruits of Rosa spp. and a tincture made from the buds of Betula spp. or roots of Potentilla erecta were used to treat kidney diseases. The cosmetics category was represented mainly by the decoction of the aerial parts or leaves of Urtica dioica and the decoction of the roots and flowers of Arctium tomentosum for washing hair. The only use that met the reliability criterion in the neurological category was the topical application of the leaves of Plantago major to alleviate headache.

\section{Veterinary area}

Twenty-three taxa belonging to 17 families were used for ethnoveterinary purposes and as fodder. Only three families were represented by more than one taxon: Asteraceae (five taxa), Betulaceae (two taxa), and Urticaceae (2 taxa); whereas the most diversely used taxa were Asteraceae (30 DUR), Urticaceae (9 DUR) and Fagaceae (6 DUR). All the uses within the veterinary area were mentioned by less than $10 \%$ of the respondents, and only six taxa (Artemisia absinthium, Urtica dioica, Taraxacum officinale, Quercus robur, Veratrum lobelianum, and Hypericum spp.) were used by more than two people; therefore the reliability criterion was met by only a small percentage of taxa (26\%). Three taxa were uniquely used in this area: Lathraea squamaria and Phleum spp. were both named by one interviewee and Veratrum lobelianum was mentioned by three persons. Seven taxa (Achillea millefolium, Alnus spp., Chelidonium majus, Equisetum arvense, Juniperus communis, Tanacetum vulgare and Trifolium spp.) overlap with taxa used for healing humans but not with those used for human food; all but the last were used by only one person.

Among the 34 people who claimed to have used wild plants in the veterinary area, the two most knowledgeable used six and three taxa, numbering eight and six DUR, respectively. Seventeen people ( $50 \%$ of all respondents who mentioned the veterinary use of wild plants) named only a single use of wild plants. The mean number of used taxa was 1.6 and the mean number of DUR was 2.0.

All the DUR in the ethnoveterinary area were distributed between 33 emic categories, which were attributed to three main general categories related to the healing of animals and to one category for fodder; $21 \%$ of DUR belonged to diverse emic categories of which not a single use met the reliability criterion (Table 4, Fig. 5).

The Informant Consensus Factor for the whole veterinary area was low 0.67 (68 use citations for 23 taxa). However, only one general veterinary category (rumination problems) had a FIC equivalent to the FIC of the area, with two taxa (Artemisia absinthium and Veratrum lobelianum) meeting the reliability criterion for this specific use. One other category, gastrointestinal, had FIC $=0.63$ (eight taxa all used against diarrhoea) with two taxa meeting reliability criterion: Artemisia absinthium for pigs and cows (and chickens) and Quercus robur for 
Table 4 General categories in veterinary area, included emic veterinary categories and Use Citations

\begin{tabular}{|c|c|c|}
\hline Food category & Emic veterinary categories & UC \\
\hline \multirow[t]{4}{*}{ Diarrhoea (20) } & Diarrhoea in calves & 2 \\
\hline & Diarrhoea in chicken & 1 \\
\hline & Diarrhoea in cows & 10 \\
\hline & Diarrhoea in pigs & 8 \\
\hline \multirow[t]{9}{*}{ Fodder (21) } & Fodder for bees in spring & 1 \\
\hline & Fodder for calves & 1 \\
\hline & Fodder for chicks & 1 \\
\hline & Fodder for cows & 3 \\
\hline & $\begin{array}{l}\text { Fodder for cows, to increase milk } \\
\text { production }\end{array}$ & 1 \\
\hline & Fodder for home animals & 2 \\
\hline & Fodder for pigs & 5 \\
\hline & Fodder for rabbits & 4 \\
\hline & Fodder for turkey & 3 \\
\hline \multirow[t]{13}{*}{ Other (14) } & Appetizer for cows & 2 \\
\hline & Bacterial diseases in bees & 1 \\
\hline & Blood in urine in cows & 1 \\
\hline & Cold in domesticated animals & 1 \\
\hline & Cuts in domesticated animals & 1 \\
\hline & Disinfectant for home animals & 1 \\
\hline & Good for cows & 1 \\
\hline & Good for horses & 1 \\
\hline & Helminthic infection in cows & 1 \\
\hline & Helminthic infection in pigs & 1 \\
\hline & $\begin{array}{l}\text { When piglets do not go to their } \\
\text { mother }\end{array}$ & 1 \\
\hline & Vitamins for cows & 1 \\
\hline & Wounds in cows & 1 \\
\hline \multirow[t]{2}{*}{ Rumination problems (7) } & Rumination problems in cows & 6 \\
\hline & Rumination problems in goats & 1 \\
\hline \multirow[t]{3}{*}{ Strengthening of animals (6) } & Strengthening of cows & 1 \\
\hline & Strengthening of piglets & 1 \\
\hline & Strengthening of pigs & 4 \\
\hline
\end{tabular}

pigs and cows. The remaining categories had relatively low Informant Consensus Factors (0.25-0.5). Among the specific taxa meeting the reliability criterion within a specific emic category were dry Urtica dioica aerial parts added to fodder to strengthen pigs and fodder for cows, Taraxacum officinale used as fodder for pigs, Artemisia absinthium as fodder for rabbits and turkey.

\section{Overlap between areas}

Figure 6 illustrates the overlap of taxa used in all three areas. Forty-seven taxa were used in both the food and

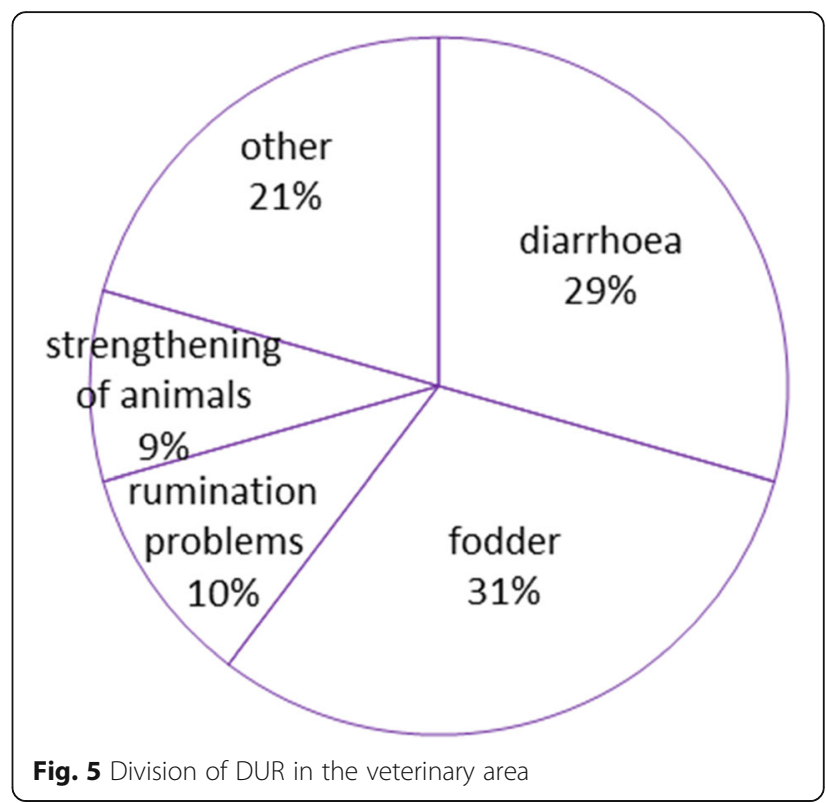

medicinal areas, which equates to an overlap of $55.3 \%$. The overlap between medicinal and veterinary areas is only $27 \%$ (20 taxa), but given that almost $87 \%$ of all taxa used in the veterinary area are also used in the medicinal area indicates that there is considerable overlap here as well (Fig. 6).

Thirty wild plant taxa had more widespread use, that is they were used by at least 14 (approximately 10\%) of the interviewees (Table 5). A comparison of the plants in the food and medicinal areas shows that the more widespread the use, the more there were people using one

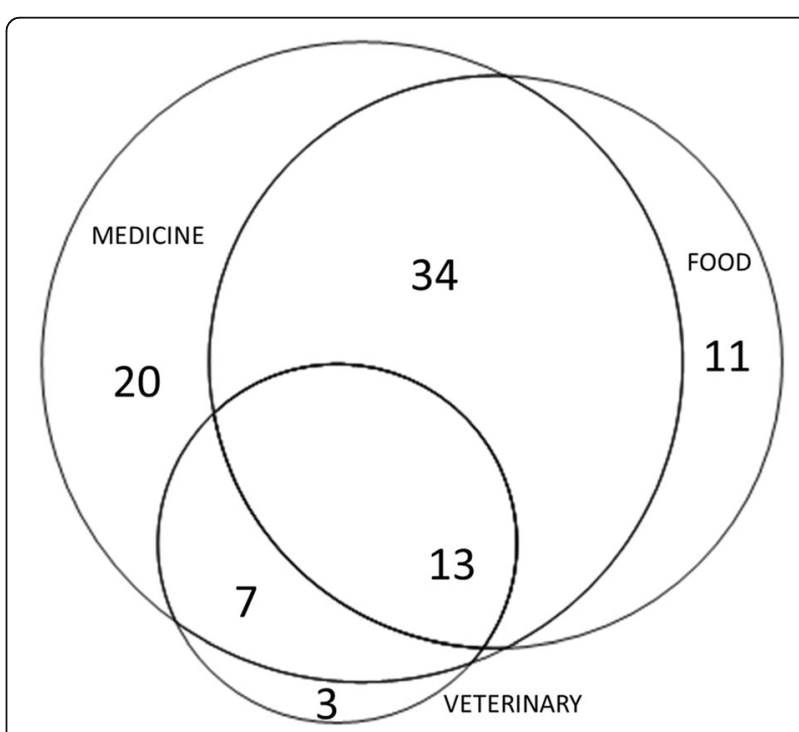

Fig. 6 Overlap between taxa used in food, medicinal and veterinary areas 
Table 5 Taxa used by at least $10 \%$ of the interveiwees

\begin{tabular}{|c|c|c|c|c|c|c|c|c|c|}
\hline Taxa & $\begin{array}{l}U \\
n=134\end{array}$ & DUR & F_U $n=124$ & F_DUR & M_U $n=113$ & M_DUR & Overlap F_U/M_U & V_U $n=34$ & V_DUR \\
\hline Vaccinium myrtillus & 108 & 224 & 95 & 169 & 41 & 55 & 28 & & \\
\hline Rubus idaeus & 85 & 170 & 69 & 108 & 33 & 62 & 17 & & \\
\hline Betula spp. & 73 & 154 & 59 & 87 & 36 & 66 & 22 & 1 & 1 \\
\hline Rumex spp. & 71 & 92 & 71 & 91 & 1 & 1 & 1 & & \\
\hline Vaccinium vitis-idaea & 64 & 117 & 54 & 91 & 18 & 26 & 8 & & \\
\hline Urtica dioica & 57 & 83 & 47 & 55 & 16 & 21 & 6 & 6 & 7 \\
\hline Tilia cordata & 52 & 73 & 34 & 35 & 28 & 38 & 10 & & \\
\hline Vaccinium oxycoccos & 51 & 77 & 48 & 66 & 8 & 11 & 5 & & \\
\hline Vaccinium uliginosum & 48 & 69 & 46 & 63 & 4 & 6 & 2 & & \\
\hline Fragaria vesca & 45 & 62 & 40 & 53 & 5 & 9 & 0 & & \\
\hline Hypericum spp. & 43 & 62 & 15 & 15 & 32 & 44 & 4 & 3 & 3 \\
\hline Chenopodium album & 41 & 41 & 41 & 41 & 0 & 0 & 0 & 1 & 2 \\
\hline Quercus robur & 40 & 66 & 30 & 35 & 19 & 25 & 9 & 5 & 6 \\
\hline Plantago major & 39 & 54 & 1 & 1 & 39 & 52 & 1 & 1 & 1 \\
\hline Armoracia rusticana & 37 & 46 & 32 & 39 & 5 & 7 & 0 & & \\
\hline Taraxacum officinale & 33 & 44 & 18 & 19 & 16 & 19 & 1 & 5 & 6 \\
\hline Viburnum opulus & 29 & 47 & 18 & 24 & 15 & 23 & 4 & & \\
\hline Potentilla erecta & 28 & 44 & 4 & 4 & 25 & 40 & 1 & & \\
\hline Rubus caesius & 28 & 39 & 28 & 37 & 1 & 2 & 1 & & \\
\hline Arctium tomentosum & 27 & 37 & 1 & 1 & 27 & 36 & 1 & & \\
\hline Allium ursinum & 22 & 28 & 21 & 27 & 1 & 1 & 0 & & \\
\hline Rosa spp. & 21 & 33 & 12 & 14 & 11 & 19 & 2 & & \\
\hline Pinus sylvestris & 20 & 43 & 7 & 10 & 15 & 32 & 2 & 1 & 1 \\
\hline Syringa vulgaris & 20 & 21 & 2 & 3 & 18 & 18 & 0 & & \\
\hline Acer platanoides & 19 & 27 & 16 & 20 & 5 & 6 & 2 & 1 & 1 \\
\hline Artemisia absinthium & 17 & 28 & 1 & 1 & 8 & 10 & 1 & 11 & 17 \\
\hline Pyrus pyraster & 16 & 31 & 11 & 13 & 11 & 16 & 6 & 2 & 2 \\
\hline Thymus serpyllum & 14 & 16 & 8 & 8 & 7 & 8 & 1 & & \\
\hline Sorbus aucuparia & 14 & 17 & 5 & 6 & 9 & 11 & 0 & & \\
\hline Tanacetum vulgare & 14 & 18 & 0 & 0 & 14 & 18 & 0 & & \\
\hline
\end{tabular}

Abbreviations: $U$ Users across areas, DUR Detailed Use Reports across areas, $F_{-} U$ Users in Food area, F_DUR Detailed Use Reports in Food area, $M \_U$ Users in Medicinal area, M_DUR Detailed Use Reports in Medicinal area, Overlap $F_{-} U / M_{-} U$ No of users who used the same taxa in both food and medicinal areas, $V \_U$ Users in Veterinary area, $V_{-} D U R$ Detailed Use Reports in Veterinary area

plant in both areas (most notably Vaccinium myrtillus, Betula spp. and Rubus idaeus), while there were specific taxa represented in both areas but predominantly used in only one, such as Rumex spp. for food and Plantago major as medicine (Fig. 7). The prevalence of plants used in the food area is clearly seen among the 20 taxa with the highest number of DUR, as medicinal use prevails in only three taxa (Plantago major, Potentilla erecta, Hypericum spp.) (Fig. 8).

\section{Used parts and modes of use}

Across all areas fruits and seeds were the most commonly used parts of a plant (36\%), followed by leaves (18\%), aerial parts (17\%), flowers and inflorescences (8\%), sap (6\%), roots (5\%) and twigs (3\%), and all other parts (7\%). In the food area, fruits alone (without seeds) had an even greater share $(48 \%)$, as did the use of sap (8\%), whereas aerial parts (11\%) and flowers with inflorescences (4\%) were less important. In the medicinal area, aerial parts and fruits dominated the list, both at $20 \%$, and the proportion of roots (8\%), twigs (6\%), buds and cones (both $3 \%$ ), and bark (2\%) was also increased compared to their distribution in all three areas combined. The veterinary area is the only area in which the use of leaves had a considerably smaller proportion than across all areas (4\%); whereas the use of aerial parts (63\%) and bark (6\%) had much greater proportions. 


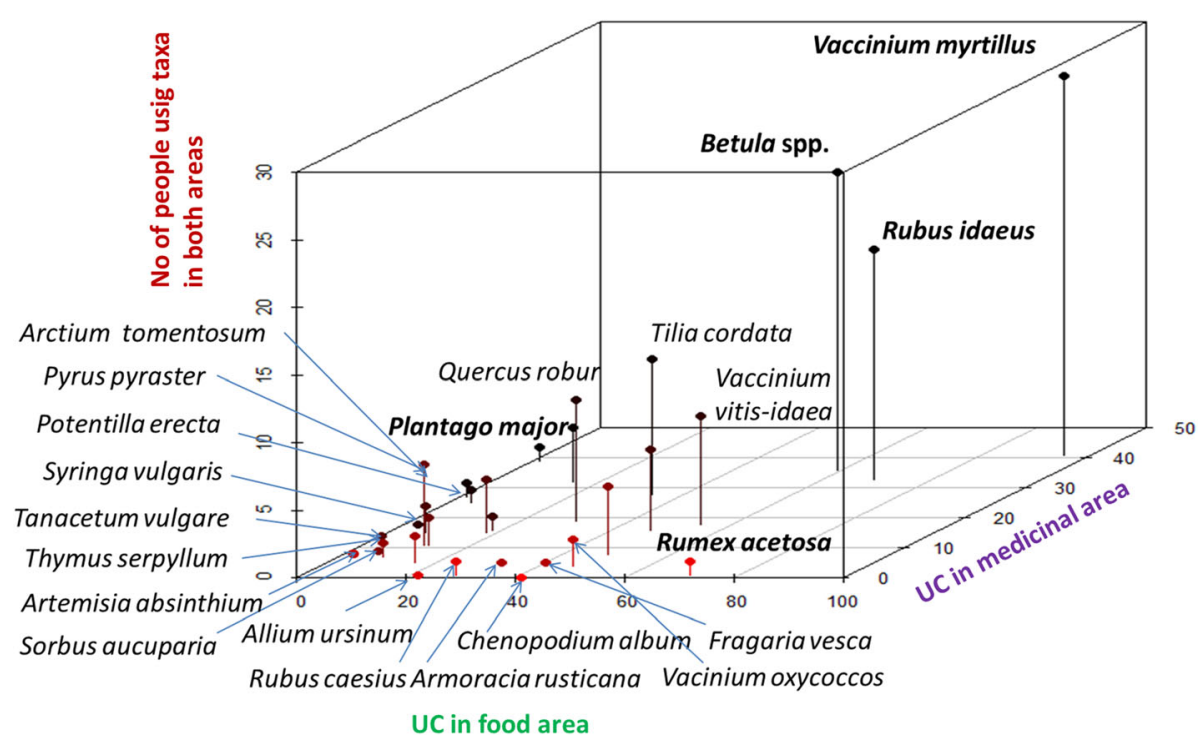

Fig. $73 \mathrm{D}$ scatter plot of the overlapping use of 30 plants mentioned by at least $10 \%$ of the interviewees in the food and medicinal areas; designed in $\mathrm{R}[65]$

The vast majority of use modes across the areas are divided among three possibilities: plants are used either fresh $(41 \%)$, as a tea or decoction $(20 \%)$, or dried $(12 \%$, including plants dried for recreational tea). In the food area, plants are primarily used fresh (70\%), and less often dried (19\%), frozen $(4 \%)$, fermented $(4 \%)$ or otherwise processed (3\%, mainly preserved for winter in the form of compotes, etc.). In the medicinal area, uses are more diverse (about 50 different use modes in contrast to less than 15 in the food area); tea is the most common mode of use (33\%), followed by the topical application of fresh plants (13\%), tincture (4\%), drunk tincture (10\%) and decoction (9\%). Fresh plants are eaten in only $6 \%$ of DUR, while $4 \%$ of DUR in the medicinal area refers to whisking in sauna. The eating of dried plants (4\%) and jams (3\%) as well as making baths (3\%, from decoctions) are also quite common. The remarkable presence (in total over 6\%) of different food categories (jam, compote,

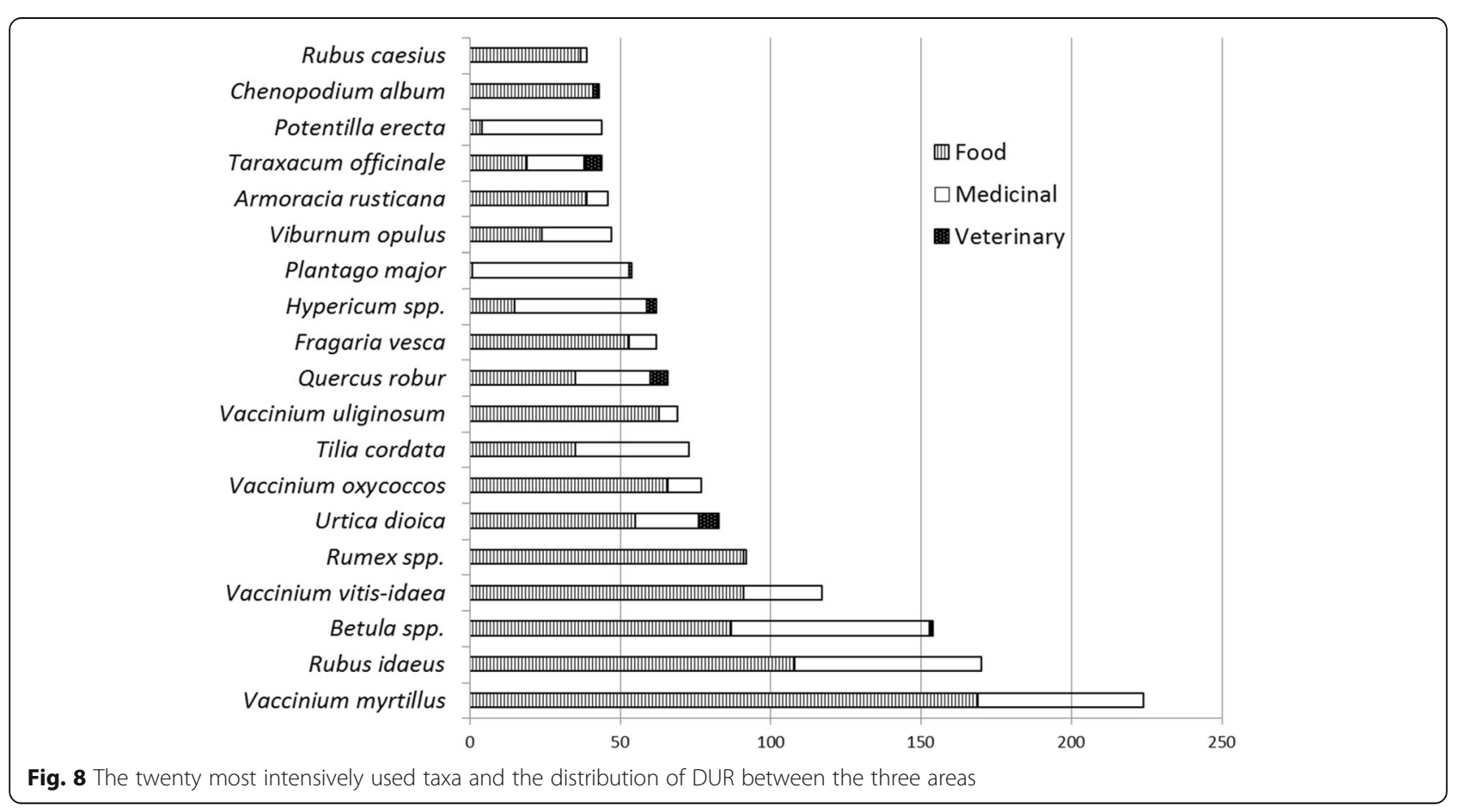


fermented sap, etc.) in the medicinal area stresses the interrelated nature of the two areas. In the veterinary area the primary use modes are decoction (38\%) and fresh (15\%, mainly fodder), as well as fresh or dried taxa added to fodder (both 12\%).

\section{Past and temporary uses}

Seventeen percent of the DUR used in the food and veterinary areas and $10 \%$ of the ones from the medicinal area were claimed to have been used only in the past (14\% across all areas). Past uses in the food area followed the pattern of general food use, with some exceptions: the most prevalent uses abandoned in the past were snacks and soups (both $25 \%$ of all past uses), followed by jams (13\%) and recreational teas (5\%). Although on a general level past uses were distributed more or less proportionally (one or two uses here and there), some past uses in the food area constituted a considerable number of the uses in specific emic categories: more than half the uses of soup made from Chenopodium album and a bit less than half the reports of soup made from Urtica dioica. In addition, two-thirds of the uses of the leaves of Quercus robur which are put under bread during baking and snacking of the leaves of Oxalis acetosella were attributed to the past.

Past uses in the medicinal area are either treatments of childhood diseases (by the mother of interviewees), or uses which respondents had abandoned in later life due to reduced access to the plant (including a decrease of personal mobility) or limited need for healing (for example, cold and some childhood diseases). The majority of past uses are related to general health (mainly prophylactics) and gastrointestinal issues, followed by the dermatological and respiratory categories. The pattern follows quite closely the general distribution of the categories in the medicinal area. Some exceptions, however, exist; for example, the past healing of wounds with leaves from Acer platanoides twigs blessed in a church on Pentecost seems to be one of the few remnants of the so-called magical thinking in the quite practical realm of modern domestic medicine in Belarus. A few other ritual practices recalled from the past include: the collecting of Vaccinium myrtillus fruits, strictly on St. John's day, and then drying and storing them apart from other fruits, to be later used to alleviate stomach ache; or wearing dried roots of Acorus calamus underneath the clothing for repelling the evil eye. In the veterinary area a few abandoned uses were related to childhood, while others to the past when domestic animals were kept. The majority of past uses, however, in both medicinal and veterinary areas are quite similar to those still used today.

The proportion of uses that were claimed as temporary was relatively small ( $1 \%$ across all areas). However, they also deserve closer attention, as there might be many more of them; a limitation of the method employed in this study is that it did not allow sufficient time for recalling temporary uses. In the food area temporary uses were related either to short-term food shortages in war time (acorns of Quercus robur as a bread additive, and fresh leaves of Urtica dioica and young cones of Pinus sylvestris as snacks), or taste trials (fermentation of Betula spp. sap, jam from young cones of Pinus sylvestris, salad made from Taraxacum officinale leaves and popular jam made from the flowers of Taraxacum officinale). Temporary medicinal uses were mostly related to the treatment of young children (the need disappeared when children got older), such as the use of fruits and twigs of Rubus idaeus to treat cold and cough, jam of Taraxacum officinale against sore throat and bronchitis, fresh fruits of Viburnum opulus against cold, leaves of Tussilago farfara against cough, aerial parts of Bidens tripartita for diathesis, and decoction of aerial parts Chelidonium majus to treat chickenpox infectiona use claimed to be prescribed by a doctor. A few uses also related to temporary illness of the interviewee or his or her relatives, such as the use of the leaves of Vaccinium vitis-idaea to treat urinary problems or the twigs of Betula spp. or Quercus robur in a prophylactic bath. One middle aged woman described a ritual for treating her son for epilepsy with the buds of Betula spp.: I went to the crossroad at three o'clock in the morning, gathered buds from nine birches; the next morning I brought nine anthills, covered them with hot water and left them in a hot place for nine days to ferment; I later bathed my son in it. Given the details of the ritual described, this seems like a historical use tried out in the time of need. However, the woman added that she read about it in a magazine and decided to try it out, and it helped, he did not perish. The majority of temporary uses are common in the regional ethnomedicine or official medicine, and in the food area, so in fact those were temporary uses only for specific interviewees.

\section{Popular attitudes towards the use of wild plants}

Quite often, while asking about the use of wild plants, we encountered answers such as: I know nothing, I am too old, I don't remember or you should ask someone who knows, we don't deal with such things. It appeared that people did not consider this kind of knowledge as something worth discussing. After further inquiry, people usually first mentioned forest fruits and all the preserves that they make with them, followed by recreational teas, sometimes trying to change the subject, and also stressing the medicinal properties of the same or other teas (sometimes just knowing the uses, but not really applying the tea for that purpose-and thus not counting as a valid record). Soups and other hot dishes 
were recalled readily, although younger people seem to be ashamed of knowing how to cook nettle soup, as its use is somehow related with poverty. While condiments constituted a remarkable category, we literally had to name all potential application possibilities (strong alcohol, preservation of cucumbers, etc.), to help people recall their uses. By the end of the interview we often noticed a shift in the attitude towards wild plants and sometimes even the realization of the importance of the subject and interviewees knowledge about it.

Men, talking about the use of wild plants, often referred to women, especially regarding food plants, but in fact there are many quite knowledgeable men as well, even if they do not prepare food or medicate family-they at least know what is done in their family. One of the tasks of men is foraging, especially for tree sap, as well as making home-made alcohol, although officially both activities are illegal. While asking about home-made vodka (and plants soaked in it), we sometimes had to refer to other villagers (without mentioning names)-after hearing that neighbours had taken pride in their moonshine and all it contains, they gladly showed their "harvest".

Some people remarked that wild plants were useful as long as they were clean, and that now radiation has taken away all their good qualities: what can you get from a plant after radiation. Others mentioned that they cultivate all they need, and that earlier (when they were younger) it was easier to just go and collect plants in the wild, but now it is easier to grow them in the garden. Yet some other people said that they never had time to collect in the wild, as work in the collective farm took all their time.

\section{Discussion}

The dominance of Rosaceae and Asteraceae is common in many parts of Europe (see [4] and the references within), and the high number of uses of Ericaceae may be due to the intensive use of forest fruits in both the food and medicinal areas, which may be peculiar to the relatively Nordic location where such taxa grow, seeing that in Estonia the use of Ericaceae is also quite widespread $[8,54]$. The low percentage of Lamiaceae, however, is surprizing and may be due to its exclusion from the sample those taxa that were cultivated for food and medicinal purposes. The overlap of the food and medicinal areas (over 55\%) is considerably larger than the nearly $40 \%$ found in a similar comparison described in [4], which can probably be explained by the absence of cultivated species in this study and the fact that we also included a wider general health category. However, such maximization of the versatility of limited resources is evident, although not emphasized, in many different post-Soviet (or post-socialist) regions (see for example $[5,6,60])$.
While it is not possible to compare the mean number of used plants per person for plants in the medicinal area, the mean number per person of wild plants used in the food area in the region (7.3) was nearly three times lower than the similar value obtained for Saaremaa, Estonia (19.9) [61], but close to the value recorded among Ukrainians living in Romania (7.7) [62]. The FIC for the wild food area (0.94) is, however, slightly higher than the value observed for Estonia (0.91) [61].

The category of salad in the food area deserves specific attention. While not very numerous (only ten taxa), and with a relatively low FIC (0.74), it contains one dominant taxa Allium ursinum which is a protected species. Also, the use of Stellaria media and Plantago major seems to be a remnant from times of food shortage.

Comparison with the only publication providing qualitative data on the recent use of wild food plants in the whole of Belarus [10] shows remarkable overlap within the uses that are numerously represented in this study. There are a considerable number of differences among the seldom mentioned taxa or uses, considering the fact that some of the previously published results were collected from the neighbouring region. This indicates that despite the homogenization of the population during the Soviet era, regional differences may still exist. The high proportion of snacks in [10] and their relatively low occurrence in this study may be attributed to the special emphasis of the questionnaires used by the authors of [10] and the particular interest of their respondents (future botanists) towards nature. However, the reason may also be related to regional cultural differences or, more likely, a considerable decrease of unintended contact with nature, as our interviewees repeatedly stressed that they rarely snacked on anything but berries in the wild.

The temporal division of uses (modest share of past uses, especially within the medicinal area) does not directly support the popular claim that people used more wild plants in the past. However, while folk history is the only applicable method for recalling past uses when there is no historical data available, it is not fully reliable. For example, it is relatively irrelevant to talk about the persistent freezing of wild fruits for preservation purposes, as deep-freezers only became available in the 1980s. However, as this time period is perceived as "long ago" by our interviewees, some of them referred to such a method of preservation as everlasting.

Uses in the medicinal area seem to be more susceptible to literary influence and more diverse in the details of use. For example, inserting the fruits of Vaccinium oxycoccos into one's ear to treat CO-intoxication is one of the few historical medicinal uses of those fruits in Estonia, first recorded in 1984 [63], which most likely originated from one shared literary source. However, while people often acknowledged that they took one or 
another medicinal uses of wild plants from newspapers or special popular magazines like "Home Doctor", they also frequently admitted that those or similar remedies were already used by their mothers or grandmothers, so the use of literature is perceived rather as a "refresher" of memory or an "official" acknowledgment of the correctness of the traditional practice (cf. [64]). Nevertheless, some uses are clearly influenced by media, such as the making of jam from Taraxacum officinale, which was popularly advertised in the 1980s (cf. [42]). The wide variety of preparation modes and used parts for the most popularly used plants signals either extensive individual experimentation within households or the past presence of a very rich and diverse traditional/regional use of wild plants which has been eroded.

\section{Conclusions}

This study examined the use of wild plants in the food, medicinal and veterinary areas within a small territory limited to one village council in Belarus. It contributes to the documentation of the local use of wild plants and to the understanding of the complex relationship between different use areas of wild plants. We discovered relatively high overlap in the taxa used in the food and medicinal areas and an even higher overlap between the medicinal and veterinary areas. As the majority of taxa with overlapping uses belonged also to the most utilized plants, there appears to be a clear tendency to use plants in several different areas once they are brought into the home. While the number of wild taxa used is relatively high, the mean number of taxa used per person is quite low, which indicates the minor importance of wild plants in the respective areas in the investigated region of Belarus.

\section{Abbreviations}

DUR: Detailed Use Records; FIC: Informant Consensus Factor; UC: Use Citations

\section{Acknowledgements}

Our special thanks go to all the study participants of the communities in the Asaviec village council, who generously shared their knowledge. The authors thank Margus Kiis, Lagle Aim and Laura Rachel Vesik for assistance in the field.

\section{Funding}

The field study was a part of an ethnobotany field school financed by the Foreign Ministry of Estonia through the Developmental Cooperation Program (66-2015-A). Research was supported by an Estonian Science Foundation Grant IUT22-5 and by the European Union through the European Regional Development Fund (Center of Excellence in Estonian Studies, CEES).

\section{Availability of data and materials}

The voice-recorded interviews as well as their transcripts are stored at the The Center for Belarusian Culture, Language and Literature Research and are available for study by request. A structured and organized version of the data is available from the first author upon reasonable request.

\section{Authors' contributions}

RS and RK designed the methodology and approach and also wrote the peer-reviewed project proposal that financed the field study. SV addressed organizational details during the fieldwork. All authors (except $\mathrm{YV}$ ) conducted field research. All authors (except RS, RK, MK, and $\mathrm{Y}$ ) transcribed interviews and conducted initial systematization. RS and RK completed the data systematization and analysed the data. RS drafted the manuscript. YH, YV, IV YK and JuP contributed to the writing of the publication, as well as composing the historiography of the subject in Belarus and a description of the region. IV and YH also contributed to the Results section (opinions) and discussion. JaP was responsible for verifying the correct phonetic spelling of local plant names. All authors read and approved the final manuscript.

\section{Competing interests}

The authors declare that they have no competing interests.

\section{Consent for publication}

Not applicable.

Ethics approval and consent to participate

As there is no special Ethics Committee for such research in Belarus, the ethical aspects of the study were approved by the The Center for Belarusian Culture, Language and Literature Research. The research adhered to the local traditions for such research and the Code of Ethics of the International Society of Ethnobiology (ISE 2008). Prior oral informed consent was obtained from all study participants.

\section{Author details}

${ }^{1}$ Estonian Literary Museum, Vanemuise 42, Tartu 51003, Estonia. ${ }^{2}$ The Center for Belarusian Culture, Language and Literature Research, Surhanava St., 1 , Bldg. 2, Minsk 220072, Belarus. ${ }^{3}$ Valožynski district, v. Vialikaja Dajnava, Padhornaja st. 118, Minsk region 222352, Belarus. ${ }^{4}$ Liuban District Culture Center, Pieršamajskaja st., 30, Liuban 223820, Belarus. ${ }^{5}$ The Belarusian State University of Culture and Arts, Rabkoraŭskaja st. 17, Minsk 220007, Belarus.

Received: 17 November 2016 Accepted: 14 January 2017

Published online: 08 February 2017

\section{References}

1. Etkin NL. Eating on the wild side. The Pharmacologic, Ecologic and Social Implications of Using Noncultigens. Tucson: University of Arizona Press; 1994

2. Etkin NL. Edible medicines: an ethnopharmacology of food. Tucson: University of Arizona Press; 2008.

3. Pieroni A, Price LL. Eating and healing: traditional food as medicine. New York: Haworth Press; 2006

4. Alarcón R, Pardo-de-Santayana M, Priestley C, Morales R, Heinrich M. Medicinal and local food plants in the south of Alava (Basque Country, Spain). J Ethnopharmacol. 2015;176:207-24.

5. Söukand R, Pieroni A. The importance of a border: Medical, veterinary, and wild food ethnobotany of the Hutsuls living on the Romanian and Ukrainian sides of Bukovina. J Ethnopharmacol. 2016:185:17-40.

6. Quave $\mathrm{CL}$, Pieroni A. A reservoir of ethnobotanical knowledge informs resilient food security and health strategies in the Balkans. Nat Plants. 2015;1:14021.

7. Sõukand R, Kalle R. Change in medical plant use in Estonian ethnomedicine: a historical comparison between 1888 and 1994. J Ethnopharmacol. 2011; 135(2):251-60

8. Sõukand R. Perceived reasons for changes in the use of wild food plants in Saaremaa, Estonia. Appetite. 2016:107:231-41.

9. Serrasolses G, Calvet-Mir L, Carrió E, D'Ambrosio U, Garnatje T, Parada M, Vallès J, Reyes-García V. A Matter of Taste: Local Explanations for the Consumption of Wild Food Plants in the Catalan Pyrenees and the Balearic Islands. Econ Bot. 2016;70:176-89.

10. Łuczaj Ł, Köhler P, Pirożnikow E, Graniszewska M, Pieroni A, Gervasi T. Wild edible plants of Belarus: from Rostafiński's questionnaire of 1883 to the present. J Ethnobiol Ethnomed. 2013;9:21.

11. Tyszkiewicz E. Opisanie powiatu Borysowskiego pod względem statystycznym, gospodarczym, przemysłowo-handlowym gospodarczym, i lekarskim, etc. Wilno: Druk Marcinowskiego; 1847.

12. Romanov YE. Belorusskiy sbornik. Vyp. 5. Vitebsk: Tip-lit. G. A. Malkina; 1891.

13. Romanov YE. Belorusskiy sbornik. Vyp. 8. Vil'no: Tip. A. G. Syrkina; 1912. 
14. Nikiforovskiy N. Ocherki prostonarodnogo zhit'ya-byt'ya $\vee$ Vitebskoy Belorussii i opisaniye predmietov obichoda. Vitebsk: Gubern. Tipo-Litograf; 1895.

15. Nikiforovskiy N. Prostonarodnye primety i povier'ya, suyevernyye obryady i obychai, legendarnye skazaniya o licakh i miestakh Vitebskoy Belorussii. Vitebsk: Gubern. Tipo-Litograf; 1897.

16. Nikiforovskiy N. Osvyashchennyye predmety i otnosheniye k nim prostonarod'ya Vitebskoy Belorussii. Vitebsk: Gubern. Tipo-Litograf; 1903.

17. Federowski M. Lud Białoruski na Rusi Litewskiej. Materiały do etnografii słowiańskiej zgromadzone w latach 1877-1905. T. 1. Kraków: Wyd-wo Komisji Antropologicznej Akademii Umiejętności; 1897.

18. Federowski M. Lud białoruski na Rusi Litewskiej: Materiały do etnografi słowianskiej zgromadzone w latach 1877-1905. T. 4. Warszawa: T-wo nauk. Warszawskie; 1935.

19. Shein P. Materialy dlya izucheniya byta i yazyka russkogo naseleniya SeveroZapadnogo kraya. T. II. Sankt-Peterburg: Tipografiya Imperatorskoy Akademii nauk; 1893.

20. Shein P. Materialy dlya izucheniya byta i yazyka russkogo naseleniya SeveroZapadnogo kraya. T. III. Sankt-Peterburg: Tipografiya Imperatorskoy Akademii nauk; 1902.

21. Dobrovol'skiy V. Smolenskiy etnograficheskiy sbornik. v 4 ch. Moskva: SanktPeterburg; 1891-903.

22. Bahdanovič A. Pieražytki staražytnaha svietasuzirannia ŭ bielarusaŭ. Etnahrafičny narys. Repryntnaje vydannie 1895. Minsk: Bielaruś; 1995.

23. Wereńko F. Przyczynek do lecznictwa ludowego. In: Materjały antropologiczno-archeologiczne i etnograficzne. T. 1. Kraków: Wyd-wo Komisji Antropologicznej Akademii Umiejętności; 1896. p. 99-228.

24. Yanchuk N. Po Minskoy gubernii. In: Sbornik svedeniy dlya izucheniya byta krest'yanskogo naseleniya Rossii. Vyp. 1. Moskva: Tip. A. Levenson i K; 1889. p. 213-21.

25. Orzeszkowa E. Ludzie i kwiaty nad Niemnem. Wisła. 1888;2:1-15. 675-703: 1890:4:1-31; 1891:5:235-247.

26. Moszyński K. Kultura ludowa słowian. T. 2, zs. 1. Warszawa: Książka i Wiedza; 1967.

27. Piatkievič Č. Rečyckaje Paliessie. Minsk: Bielaruski knihazbor; 2004.

28. Zelenin D. Totemicheskiy kul't derevv'yev u russkikh i u belorusov. Izvestiya Akademii Nauk SSSR: Otdelenie obshestvennykh nauk, 1933;6:591-629.

29. Popov I. Belorussiya i belorusy. Moskva: M.V. Klyukin; 1912

30. Min'ko L. Narodnaya medicina i vred znakharstva. Minsk: n.p.; 1962.

31. Min'ko L. Narodnaya medicina Belorussii (Kratkiy istoricheskiy ocherk). Minsk: Nauka i tekhnika; 1969

32. Švied I. Raslinnyja simvaly bielaruskaha fálkloru. Brest: Vydaviectva BrDU; 2000.

33. Valodzina T. Miesca pravilaŭ u kaliandarnaj miedycynskaj mahii. In: Valodzina T, Hurski A, Bartaševič $H$, Kabašnikaŭ K, editors. Malyja žanry. Dziciačy faĺklor. Minsk: Bielaruskaja navuka; 2004. p. 68-88.

34. Valodzina T. Miforitual'nyj i etnolingvisticheskiy aspekty narodnoy mediciny belorusov: epilepsiya. Studia Litteraria Polono-Slavica: Morbus, medicamentum et sanus-Choroba, lek i zdrowie. 2001;6:99-108

35. Valodzina T. Ciela čalavieka: slova, mif, rytual. Minsk: Technalohija; 2009.

36. Volodina T. Iz narodnoy mediciny belorusov. In: Zhivaya starina. vol 4. 2004. p. 37-8.

37. Volodina T. Narodnaja miedycyna: rytuaĺna-mahičnaja praktyka. Minsk: Bielaruskaja navuka; 2007.

38. Lobač U. Eliemienty kulturnaj prastory ŭ sistemie narodnaj miedycyny Liepielščyny. In: Liepielskija čytanni. Liepiel: n.p.; 2004. p. 18-22.

39. Filipienka U. Anatama-fizijalahičnyja ŭjaŭlienni, etyjalohija i prafilaktyka chvarob u sistemie tradycyjnaj narodnaj miedycyny bielarusaŭ Padzvinnia. Vestnik Polotskogo gosudarstvennogo universiteta. Gumanitarnyye nauki. 2007;1:26-35.

40. Cruz-Garcia GS, Price LL. Human-induced movement of wild food plant biodiversity across farming systems is essential to ensure their availability. J Ethnobiol. 2014;34:68-83.

41. Cruz-Garcia GS, Price LL. Ethnobotanical investigation of 'wild' food plants used by rice farmers in Kalasin, Northeast Thailand. J Ethnobiol Ethnomed. 2011;7:33.

42. Łuczaj L, Pieroni A, Tardío J, Pardo-de-Santayana M, Sõukand R, Svanberg I, Kalle R. Wild food plant use in 21st century Europe: the disappearance of old traditions and the search for new cuisines involving wild edibles. Acta Soc Bot Pol. 2012:8:359-70
43. Menendez-Baceta G, Aceituno-Mata L, Tardío J, Reyes-García V, Pardo-deSantayana M. Wild edible plants traditionally gathered in Gorbeialdea (Biscay, Basque Country). Genet Resour Crop Evol. 2012;59:1329-47.

44. Respublika Belarus'. Entsiklopediya: V 6 t. T. 1. Minsk: BelEn; 2005.

45. Priroda Belarusi. Entsiklopediya. V 3 t. T. 3. Rasteniya, griby, zhivotnyye. Minsk: Belaruskaya entsyklapedyya; 2014.

46. Harady i vioski Bielarusi. Encyklapiedyja. T. 8: Minskaja voblasć. kn. 3. Minsk: Bielaruskaja Encyklapiedyja imia Pietrusia Broŭki; 2012.

47. Hudson C. Folk history and ethnohistory. Ethnohistory. 1966;1:52-70.

48. ISE. The ISE (International Society of Ethnobiology) Code of Ethics. 2008. Available at: http://www.ethnobiology.net/what-we-do/core-programs/iseethics-program/code-of-ethics/. Accessed 17 Oct 2015.

49. Tutin T, Heywood V, Burges N, Valentine D, Walters S, Webb D. Flora Europaea. Cambridge, UK: University Press; 1964.

50. Stevens PF. Angiosperm Phylogeny Website, version 13. 2012. Available at: http://www.mobot.org/MOBOT/research/APweb/. Accessed 01 Oct 2016

51. The Plant List. Version 1.1. Published on the Internet. 2013. Available at: http://www.theplantlist.org/. Accessed 24 Aug 2016.

52. Sõukand R, Kalle R. Where does the border lie: locally grown plants used for making tea for recreation and/or healing, 1970s-1990s Estonia. J Ethnopharmacol. 2013;150:162-74.

53. Łuczaj Ł, Kujawska M. Botanists and their childhood memories: an underutilized expert source in ethnobotanical research. Bot J Linn Soc. 2012;168:334-43.

54. Kalle R, Sõukand R. Wild plants eaten in childhood: a retrospective of Estonia in the 1970s-1990s. Bot J Linn Soc. 2013;172(2):239-53.

55. Trotter RT, Logan MH. Informant consensus: a new approach for identifying potentially effective medicinal plants. In: Etkin NL, editor. Plants in Indigenous Medicine and Diet, Behavioural Approaches. New York: Redgrave Publishing Company; 1986. p. 91-112.

56. Johns T, Kokwaro JO, Kimanani EK. Herbal remedies of the Luo of Siaya District, Kenya: Establishing quantitative criteria for consensus. Econ Bot. 1990;44:369-81.

57. Sõukand R, Quave CL, Pieroni A, Pardo-de-Santayana M, Tardío J, Kalle R, Łuczaj Ł, Svanberg I, Kolosova V, Aceituno-Mata L, Menendez-Baceta G. Plants used for making recreational tea in Europe: a review based on specific research sites. J Ethnobiol Ethnomed. 2013;9:58.

58. Sõukand R, Kalle R. Changes in the Use of Wild Food Plants in Estonia 18th21st Century. New York: Springer; 2016.

59. Sõukand R, Kalle R. Perceiving the Biodiversity of Food at Chest-height: use of the Fleshy Fruits of Wild Trees and Shrubs in Saaremaa, Estonia. Hum Ecol. 2016;2(44):265-72.

60. Pieroni A, Soukand R, Quave CL, Hajdari A, Mustafa B. Traditional food uses of wild plants among the Gorani of South Kosovo. Appetite. 2017;108:83-92.

61. Kalle R, Sõukand R. Current and remembered past uses of wild food plants in Saaremaa, Estonia: changes in the context of unlearning debt. Econ Bot. 2016;70(3):235-53

62. Łuczaj Ł, Stawarczyk K, Kosiek T, Pietras M, Kujawa A. Wild food plants and fungi used by Ukrainians in the western part of the Maramureş region in Romania. Acta Soc Bot Pol. 2015;84:339-46.

63. Sõukand R, Kalle R. Historistlik eesti rahvameditsiini botaaniline andmebaas (HERBA) [HERBA, the Estonian folk medicine database of plant use]. Tartu: Estonian Literary Museum; 2008. http://herba.folklore.ee. Accessed 15 Oct 2016

64. Kołodziejska-Degórska I. Patients' webs of relations in the medical landscapes of Central Ukraine. Anthropol Med. 2016;23:155-71.

65. R Development Core Team. R: A language and environment for statistical computing. Vienna: R Foundation for Statistical Computing; 2012. http:// www.R-project.org/. 